\title{
Histone methyltransferase G9a protects against acute liver injury through GSTP1
}

\author{
Yu Zhang ${ }^{1}$ - Weili Xue ${ }^{2}$. Wenquan Zhang $\mathbb{1}^{2} \cdot$ Yangmian Yuan ${ }^{2} \cdot$ Xiuqin Zhu ${ }^{2}$ - Qing Wang ${ }^{2} \cdot$ Yujuan Wei $^{1}$. \\ Dong Yang ${ }^{1} \cdot$ Chen Yang ${ }^{1} \cdot$ Yan Chen ${ }^{1} \cdot$ Yu Sun ${ }^{2} \cdot$ Shun Wang ${ }^{3} \cdot$ Kun Huang $^{1} \cdot$ Ling Zheng $^{2}$
}

Received: 14 March 2019 / Revised: 1 August 2019 / Accepted: 16 August 2019 / Published online: 12 September 2019

(c) The Author(s) 2019. This article is published with open access

\begin{abstract}
Acute liver injury is commonly caused by bacterial endotoxin/lipopolysaccharide (LPS), and by drug overdose such as acetaminophen (APAP). The exact role of epigenetic modification in acute liver injury remains elusive. Here, we investigated the role of histone methyltransferase G9a in LPS- or APAP overdose-induced acute liver injury. Under Dgalactosamine sensitization, liver-specific G9a-deficient mice (L-G9a ${ }^{-1-}$ ) exhibited $100 \%$ mortality after LPS injection, while the control and L-G9a ${ }^{+-}$littermates showed very mild mortality. Moreover, abrogation of hepatic G9a or inhibiting the methyltransferase activity of G9a aggravated LPS-induced liver damage. Similarly, under sublethal APAP overdose, L-G9a ${ }^{-/-}$mice displayed more severe liver injury. Mechanistically, ablation of G9a inhibited H3K9me1 levels at the promoters of Gstp1/2, two liver detoxifying enzymes, and consequently suppressed their transcription. Notably, treating L-G9a ${ }^{-/-}$mice with recombinant mouse GSTP1 reversed the LPS- or APAP overdose-induced liver damage. Taken together, we identify a novel beneficial role of G9a-GSTP1 axis in protecting against acute liver injury.
\end{abstract}

\section{Introduction}

As the main detoxifying organ, liver shows remarkable capacity against bacterial/viral infection and drug toxicity, thus playing a central role in the regulation of body

These authors contributed equally: Yu Zhang, Weili Xue

Edited by C. Borner

Supplementary information The online version of this article (https:// doi.org/10.1038/s41418-019-0412-8) contains supplementary material, which is available to authorized users.

Kun Huang

kunhuang@hust.edu.cn

$\triangle$ Ling Zheng

lzheng@whu.edu.cn

1 Tongji School of Pharmacy, Tongji Medical College, Huazhong University of Science and Technology, Wuhan 430030 Hubei, PR China

2 Hubei Key Laboratory of Cell Homeostasis, College of Life Sciences, Wuhan University, Wuhan 430072 Hubei, PR China

3 Department of Blood Transfusion, Wuhan Hospital of Traditional and Western Medicine, Wuhan 430022, PR China homeostasis [1]. Acute liver injury is a serious health issue with high morbidity and mortality [2]. Bacterial lipopolysaccharide (LPS), also termed endotoxin, is known to cause acute liver injury and sepsis [3]. Drug toxicity is another leading cause of acute liver injury, overdose of nonprescription analgesic acetaminophen (APAP) accounts for more than $50 \%$ of all acute liver failure cases in USA [2].

LPS- and APAP overdose-induced acute liver failure share similar pathological mechanisms. Upon LPS stimulation, immune cells are recruited to the liver to produce proinflammatory cytokines, thus triggering inflammatory response; consequently, elevated inflammation-associated molecules are found in LPS-injured livers [3]. Similarly, a substantial number of neutrophils infiltrate the liver after APAP overdose [4]. Inflammation/reactive nitrogen species (RNS)/reactive oxygen species (ROS)-induced cell death also plays pathogenic roles in LPS/APAP-induced acute liver injury [4]. Pathological production of large amount of nitric oxide (NO) by inducible NO synthase (iNOS) leads to the formation of RNS, which further nitrates proteins to exacerbate inflammation and tissue injury [4]. Besides leading to hepatocyte death, ROS-caused oxidative stress also aggravates inflammatory responses through promoting the infiltration and activation of immune cells, and consequently contributes to the pathogenesis of LPS/APAP-induced acute liver injury [5]. 
G9a, a ubiquitously expressed histone methyltransferase encoded by EHMT2 (euchromatic histone lysine $\mathrm{N}$-methyltransferase 2), is a member of the SET domaincontaining $\mathrm{Su}$ (var)3-9 family [6]. G9a and its related protein GLP (G9a-like protein, encoded by EHMT1) catalyze mono- and di-methylation on histone H3K9 (H3K9me1 and $\mathrm{H} 3 \mathrm{~K} 9 \mathrm{me}$ ), which are respectively associated with transcriptional activation and repression. Currently, most functional studies focus on G9a-mediated H3K9me2, whereas the roles of $\mathrm{G} 9 \mathrm{a}$-mediated $\mathrm{H} 3 \mathrm{~K} 9 \mathrm{me} 1$ are less reported [7]. G9a plays important roles in diverse cellular processes, including proliferation, differentiation, senescence, and replication [7]. However, the role of G9a in acute liver injury remains unclear.

Here, using liver-specific G9a-deficient (L-G9a ${ }^{-/-}$) mice, we found abrogation of G9a exacerbated LPS- or APAP overdose-induced liver injury through increasing immune cells infiltration and RNS/ROS production, aggravating DNA damage and hepatocyte death. We further identified that the downregulation of glutathione S-transferase $\mathrm{Pi}$ (GSTP), the liver detoxifying enzyme [8], was, at least, partially responsible for the exacerbated liver injury, since recombinant GSTP1 effectively suppressed the LPS- or APAP overdose-induced liver damage in $\mathrm{L}-\mathrm{G} 9 \mathrm{a}^{-1-}$ mice. These results suggest G9a-GSTP1 axis as a potential therapeutic target for acute liver injury and associated diseases.

\section{Materials and methods}

\section{Animals}

Male C57BL/6 mice were obtained from the Center for Animal Experiment/Animal Biosafety Level-III Laboratory of Wuhan University. Breeding pairs of B6.Cg-Tg (Alb-cre) 21Mgn mice (Alb-Cre) were obtained from the Jackson Laboratory. Floxed G9a (G9a ${ }^{\text {flox/flox }}$ ) mice with C57BL/6 background were generated by the Model Animal Research Center of Nanjing University using standard protocols. Briefly, the targeting vector was designed to introduce a Neo cassette in intron 21 and LoxP sites in intron 7 and 21 of Ehmt2. Embryonic stem (ES) cells were electroporated with this vector and clones with correct insertion were identified by PCR and southern blot analyses. Correct ES cells were injected into C57BL/6 blastocysts to create chimeric mice, which were bred to obtain germ-line transmission of targeted G9a allele. G9a ${ }^{\text {flox/flox }}$ mice were first crossed with Alb-Cre mice, and the heterozygous offspring carrying Alb-Cre transgene $\left(\mathrm{G} 9 \mathrm{a}^{\text {flox/wt }}-\mathrm{Cre}\right)$ were then intercrossed to generate homozygous mice carrying Alb-Cre transgene (G9a $\left.\mathrm{a}^{\text {flox/flox }}-\mathrm{Cre}\right)$, which were used as liver-specific G9a knockout (L-G9a ${ }^{-1-}$ ) mice. Other littermates, G9a ${ }^{\text {wt/wt }}-$ Cre or mice carrying no Alb-Cre transgene were used as the controls
(Con). Mice were housed in ventilated microisolator cages with free access to water and regular chow. Sample sizes, as described in figure legends, were selected based on effect size and availability as per usual standard. Sample sizes $(n)$ indicated in figure legends refer to the number of animal. Randomization was done by selecting animals of similar age and weight. Blinding was involved in histological studies. Animals were handled according to the Guidelines of the China Animal Welfare Legislation, as approved by the Committee on Ethics in the Care and Use of Laboratory Animals of College of Life Sciences, Wuhan University.

\section{Genotyping}

Genotyping was performed by PCR assay using genomic DNA obtained from mouse tail. The primer set $5^{\prime}$-TGT GAGTTCCAGGTAGTGGC-3' and 5'-GAATGCCACAC AGCAGTGAC-3' were used to detect the wildtype allele (229 bp) and the G9a LoxP allele (347 bp) (Fig. S1A). Mice carrying Alb-Cre transgene were detected by the primer set 5'-GAACCTGATGGACATGTTCAGG-3' and 5'-AGTGC GTTCGAACGCTAGAGCCTGT-3'.

\section{LPS, D-galactosamine (D-Gal), BIX01294 (BIX), and APAP injection}

LPS (E. coli, 0111:B4) and D-Gal were purchased from Sigma (St Louis, MO), and APAP was obtained from Macklin (Shanghai, China). BIX, a specific G9a methyltransferase activity inhibitor [9], was obtained from Targetmol (Boston, MA). For D-Gal and LPS co-treatment, male mice were intraperitoneally (i.p.) injected with 200 $\mathrm{mg} / \mathrm{kg} \mathrm{BW}$ (body weight) of $\mathrm{D}-\mathrm{Gal}$ and $3 \mathrm{mg} / \mathrm{kg} \mathrm{BW}$ of LPS, or same volume of vehicle, and mortality was recorded within $36 \mathrm{~h}$ (Fig. S1B). Liver samples were collected either immediately from the dead mice, or from surviving mice at $36 \mathrm{~h}$ after co-injection. Blood samples were collected from tail vein of all surviving mice at $16 \mathrm{~h}$ after coinjection. For LPS-only injection, male and female mice were i.p. injected with a single dose of LPS (3 mg/kg BW) or same volume of vehicle (Fig. S1C). For BIX and LPS cotreatment, C57BL/6 male mice were i.p. injected with BIX (50 mg/kg BW) for 2 successive days. On day 2, LPS ( $3 \mathrm{mg} / \mathrm{kg} \mathrm{BW}$ ) was given after BIX injection, while mice injected with vehicle or BIX or LPS alone were used as the controls (Fig. S1D). For APAP overdose-induced acute liver injury, male mice were i.p. injected with APAP (400 $\mathrm{mg} / \mathrm{kg} \mathrm{BW}$ ) or same volume of vehicle after overnight fasting (Fig. S1E). At 1 or $24 \mathrm{~h}$ after LPS/APAP injection, serum and livers were collected. Histological evaluation and western blots were performed at $24 \mathrm{~h}$ after LPS or APAP injection; while quantitative real-time PCR (qPCR) was performed at 1 or $24 \mathrm{~h}$ after injection. 
Cell culture, primary hepatocyte isolation, plasmid construction, transfection/infection, and BIX treatment

HepG2 (human hepatocellular carcinoma cell line) cells and Hepa1-6 (murine hepatoma cell line) cells, which were purchased from Procell (Wuhan, China) and Rochen (Shanghai, China), respectively. Cells were cultured at $37{ }^{\circ} \mathrm{C}$ in a $5 \% \mathrm{CO}_{2}$ incubator, and maintained in DMEM (Hyclone, South Logan, UT, USA) supplemented with $10 \%$ FBS (Hyclone) and 1\% penicillin-streptomycin (Hyclone). Primary mouse hepatocytes were isolated as previously reported [10]. In brief, liver was perfused with calcium-free solution and then digested with collagenase (Sigma, St. Louis, MO) perfusion. Dispersed cells were released, and hepatocytes were collected and plated in collagen-coated plates with DMEM media plus 10\% FBS. Plates were washed $4 \mathrm{~h}$ later to remove non-adherent cells, and the adherent hepatocytes were used for experiments.

Two short hairpin RNAs targeting human G9a sequence, shG9a-1 and shG9a-2 (5'-GGTTTGCGCTTCAACTCAA$3^{\prime}$ and 5'-GGCGATTGCTCCAGGAATT-3', respectively), were cloned into the pSUPER plasmid. HepG2 cells were transfected with shScram (containing a scrambled nontargeting sequence) or shG9a plasmid by using TurboFect transfection reagent (Thermo Scientific, Rockford, IL), and stable knockdown cells were selected with $1 \mu \mathrm{g} / \mu \mathrm{l}$ puromycin (Amresco, Solon, OH).

Two systems were used to overexpress mouse G9a in primary hepatocytes. PAdeno-MCMV-EGFP-P2A-G9a$3 *$ Flag (Ad-G9a) and control plasmids were constructed, packed into adenovirus, and purified (Obio, Shanghai, China). Primary hepatocytes were infected with G9a or control adenovirus at an MOI of 50 for $6 \mathrm{~h}$. Alternatively, primary hepatocytes were transfected by pCAGGS-mG9a or control vector with lipofectamine 2000 (Invitrogen, Grand Island, NE). Cells was collected at $48 \mathrm{~h}$ after infection or transfection. For BIX (Cayman, Ann Arbor, MI) treatment, HepG2 cells or primary hepatocytes were treated with different dosages of BIX $(0,2,4$, or $8 \mu \mathrm{M})$ for $24 \mathrm{~h}$. All the experiments were repeated at least three times with 2-3 samples per group at each time.

\section{Preparation and administration of recombinant GSTP1 protein (rGSTP1)}

Mouse Gstpl cDNA was amplified by PCR and cloned into pET-28a, which contains an N-terminal His-tag. rGSTP1 was expressed in E. Coli and purified using $\mathrm{Ni}^{2+}$-NTA agarose (Qiagen, Valencia, CA) as previously described [11]. L-G9a ${ }^{-1-}$ mice were intravenously (i.v.) injected with rGSTP1 $(10 \mathrm{mg} / \mathrm{kg})$ or vehicle at $30 \mathrm{~min}$ prior to LPS or
APAP injection, and sacrificed at $24 \mathrm{~h}$ after the injection (Fig. S1F-G).

\section{Western blots and quantitative real-time PCR (qPCR)}

Tissues were sonicated in ice-cold RIPA buffer (Beyotime, China) and protein concentrations were determined. Total RNA was extracted using RNAiso Plus (TaKaRa Biotechnology, Japan). Targeted protein expression levels were quantitated relative to the internal control in the same sample, and then normalized to the respective control group, which was arbitrarily set as 1. qPCR was performed with $\beta$-actin as the internal control, with the relative difference of targeted gene expressed as fold change calculated by the $2^{-\Delta \Delta \mathrm{CT}}$ method. Antibodies and primers used were listed in Table S1.

\section{Protein identification by mass spectrometry (MS)}

After SDS-PAGE and coomassie brilliant blue staining, gel pieces at target positions in each group were cut, subjected to in-gel digestion, and analyzed with a $\mathrm{Q}$ Exactive HF mass spectrometer coupled with an Easy-nLC 1000 system (Thermo Scientific, Rockford, IL). The MS data were processed using Thermo Proteome Discoverer software. MS spectra were searched by the SEQUEST algorithm against SwissProt database of mouse. The mass tolerances for precursor and fragment ions were set to $10 \mathrm{ppm}$ and $0.02 \mathrm{Da}$, respectively. Search results were filtered to $1 \%$ false discovery rate using the target-decoy strategy on both peptide and protein levels.

\section{Serum alanine aminotransferase (ALT) and aspartate aminotransferase (AST) measurements}

Serum ALT and AST levels were measured by an ADVIA 2400 Chemistry System (Siemens, Tarrytown, NY) with reagents purchased from DiaSys (DiaSys Diagnostic Systems, Shanghai, China) and Kehua (Kehua Bio-engineering, Shanghai, China), respectively.

\section{Determination of glutathione (GSH) level}

Liver GSH levels were measured by a glutathione assay kit (Beyotime) following the manufacturer's instructions. Absorbance at $412 \mathrm{~nm}$ was recorded and GSH levels were calculated from a standard curve.

\section{Histological analysis, immunochemistry staining, and TUNEL assay}

Liver tissues were collected and routinely embedded into paraffin. Liver sections were stained with hematoxylin and 
eosin (H\&E) or prepared for immunochemistry staining and TUNEL assay. For immunochemistry staining, after quenching the endogenous peroxidases with $3 \% \mathrm{H}_{2} \mathrm{O}_{2}$ and blocking unspecific binding with $2 \%$ bovine serum albumin (Amresco, Solon, OH), sections were stained with F4/80, Ly-6G, CD3, 3-nitrotyrosine, 8-oxoG, or Nrf2 (Table S1) overnight at $4{ }^{\circ} \mathrm{C}$. After extensive washing, sections were incubated with respective biotinylated secondary antibodies. Positive staining was visualized using DAB Substrate (Cwbiotech, Beijing, China) following the ABC Kit (Vector Laboratories, Burlingame, CA). For TUNEL assay, apoptotic cells were detected using a TUNEL Bright Green Apoptosis Detection Kit (Vazyme Biotech, Nanjing, China) according to the manufacturer's instructions. Positive stained areas were quantified and positive cells were counted using the Image-Pro Plus software in 4-6 fields randomly selected for each sample.

\section{Chromatin immunoprecipitation (ChIP) assay}

ChIP assay was performed as we previously described [12]. Briefly, liver tissues were minced and crosslinked with $1 \%$ formaldehyde, then quenched with $125 \mathrm{mM}$ glycine. After washed with cold PBS, samples were resuspended with digestion buffer plus $1 \mathrm{mM}$ PMSF. Crosslinked chromatin was sheared with Micrococcal Nuclease (New England Biolabs, Beverly, MA) and sonication. Chromatin was immunoprecipitated using anti-H3K9me1, anti-H3K9me2, and anti-G9a (all from Abcam, Cambridge, MA) or respective IgGs (Sigma, MO; or Santa Cruz, CA). The immune-complexes were captured with Protein $\mathrm{G}$ agarose beads (GE Healthcare, Chicago, IL). ChIP-enriched and input DNA were extracted and analyzed by qPCR, with the inputs as the internal control. Primers for ChIP assay were provided in Table S1. Regions of GSTP1/2 promoter used for ChIP assay were ranged from $-2000 \mathrm{bp}$ to TSS (transcription start site), which was downloaded from http:// genome.ucsc.edu/cgi-bin/hgNear. This region was averagely divided into four parts to cover the promoter area by one set of primer per $500 \mathrm{bp}$, and the primers (Table S1) were randomly but not selectively designed by Primer 5 .

\section{Detection of intracellular rGSTP1 by confocal microscopy}

To detect the intracellular transport of the rGSTP1, we labeled rGSTP1 with fluorescein isothiocyanate (FITC) to treat the Hepa1-6 cells. Briefly, cells were starved for $12 \mathrm{~h}$ before being treated with or without inhibitors of endocytosis (chlorpromazine hydrochloride (CPZ; Targetmol, Washington, MA; $20 \mu \mathrm{M}$ ), dynasore (Targetmol; $50 \mu \mathrm{M}$ ) or $\mathrm{M} \beta \mathrm{CD}$ (Targetmol; $0.5 \mathrm{mM}$ )) for $1 \mathrm{~h}$, followed by $1 \mathrm{~h} \mathrm{co-}$ incubation with FITC or FITC-GSTP1 $(1 \mu \mathrm{M})$. Images were taken under a ZEISS 780 confocal microscope (ZEISS, Oberkochen, Germany).

\section{Statistical analysis}

The data were expressed as average \pm standard deviation (SD). Statistical significance was determined by analyzing the data with the nonparametric Kruskal-Wallis test followed by the Mann-Whitney test for comparison of three or more than three groups, or with the Mann-Whitney test only for comparison of two groups. Differences were considered statistically significant at $p<0.05$.

\section{Results}

\section{Hepatic G9a deletion is fatal upon LPS/D-Gal injection}

Significantly downregulated Ehmt2/G9a and H3K9me1/ me2 levels, but not Ehmt1/GLP, were observed in livers of liver-specific G9a-deficient (L-G9a ${ }^{-1-}$ ) mice (Fig. 1a, b). No significant difference was found on Ehmt2/G9a and $\mathrm{H} 3 \mathrm{~K} 9 \mathrm{me} 1 / \mathrm{me} 2$ levels between the livers of heterozygous L-G9a $\mathrm{a}^{+/-}$and control mice (Fig. 1a, b), possibly due to the random monoallelic gene expression [13]. Meanwhile, the abundance of Ehmt2/G9a, Ehmt1/GLP, and H3K9me1/me2 was similar in the muscle of control, L-G9a ${ }^{+-}$and L-G9a $\mathrm{a}^{-/-}$mice (Fig. 1a, b). Moreover, the levels of GLP were not significantly altered in shG9a stable HepG2 cells (Fig. S2A, B). Contrary to the embryonic lethal phenotype of whole-body G9a knockout mice [14], L-G9a ${ }^{-1-}$ mice were viable and fertile with no obvious abnormality.

LPS/D-Gal co-injection is a classical way to induce acute liver injury, in which $\mathrm{D}-\mathrm{Gal}$ increases the sensitivity of rodents to LPS-induced hepatotoxicity [15]. All LPS/D-Gal co-treated L-G9a $\mathrm{a}^{-1-}$ mice died within $27 \mathrm{~h}$, whereas the L-G9a $\mathrm{a}^{+/-}$and control mice showed very mild mortality with no significant difference between two groups within $36 \mathrm{~h}$ (Fig. 1c). H\&E staining was conducted, compared with the $\mathrm{L}-\mathrm{G} 9 \mathrm{a}^{+/-}$and control mice, the livers of $\mathrm{L}-\mathrm{G} 9 \mathrm{a}^{-/-}$mice exhibited severe hemorrhage, swollen hepatocytes and larger injured area (Fig. 1d). Moreover, serum ALT and AST levels, two clinical parameters of liver injury, were significantly increased in $\mathrm{L}-\mathrm{G} 9 \mathrm{a}^{-1-}$ mice compared with those of the L-G9a ${ }^{+/-}$and control mice under LPS/D-Gal coinjection, while the levels were similar among three groups without stress (Fig. 1e). More severe F4/80 staining was found in livers of $\mathrm{L}-\mathrm{G} 9 \mathrm{a}^{-1-}$ mice compared with those of the L-G9a ${ }^{+/-}$and control mice under LPS/D-Gal coinjection (Fig. 1f). These results indicated that under $D^{-}$ Gal sensitization, LPS induced a fatal acute liver injury in L-G9a ${ }^{-1-}$ mice. Since L-G9a ${ }^{+/-}$mice showed comparable 

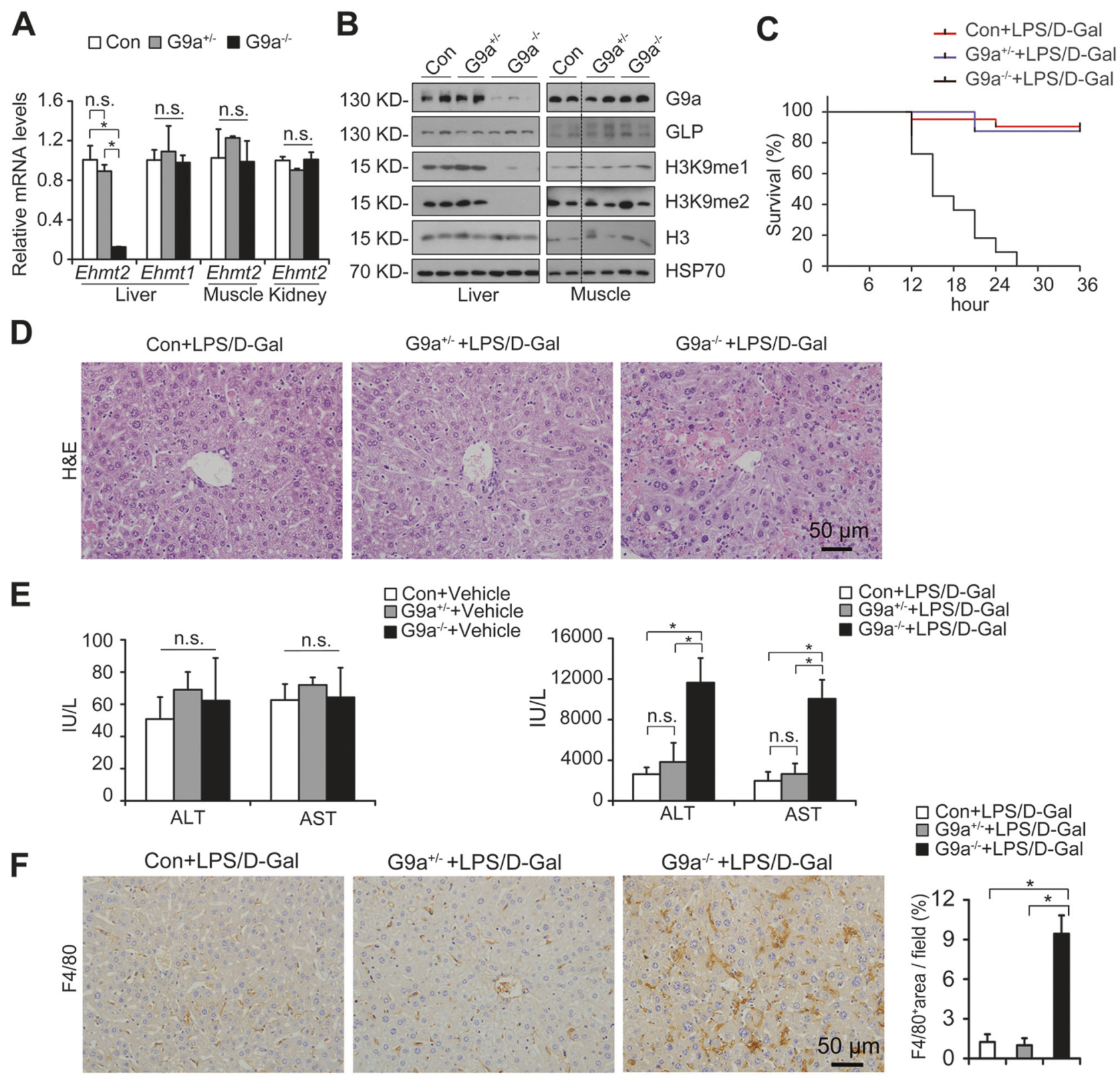

Fig. 1 Hepatic G9a deletion aggravates LPS-induced liver damage. a Relative mRNA levels of Ehmt2 and Ehmt1 in the liver, muscle and kidney of Control, L-G9a ${ }^{+/-}$and L-G9a ${ }^{-1-}$ mice. b Representative western blots for G9a, GLP, H3K9me1, H3K9me2, H3, and HSP70 in the liver and muscle of Control, L-G9a ${ }^{+1-}$ and L-G9a ${ }^{-1-}$ mice. c Survival rate $\left(n=21\right.$ for Controls; $n=16$ for $\mathrm{L}-\mathrm{G} 9 \mathrm{a}^{+/-}$mice; $n=11$

responses to LPS/D-Gal as the controls, only L-G9a ${ }^{-1-}$ and control mice were used in following studies.

\section{Hepatic G9a deletion augments LPS-induced immune cells infiltration and RNS/ROS production}

To study the role of G9a in LPS-induced acute liver injury while avoiding significantly mouse death, LPS was used alone. Consistently, histological examination revealed more for L-G9 $\mathrm{a}^{-1-}$ mice) and $\mathbf{d} \mathrm{H} \& \mathrm{E}$ staining ( $n=6-8$ per group; scale bar, $50 \mu \mathrm{m})$ after LPS/D-Gal co-injection. e Serum levels of ALT and AST with or without LPS/D-Gal co-injection. f Representative pictures for F4/80 staining in livers (left) and quantitative results (right) after LPS/ D-Gal co-injection (scale bar, $50 \mu \mathrm{m})$. All data were obtained from male mice. $n=3-4$ per group; n.s., not significant; ${ }^{*} p<0.05$

aggravated immune cells infiltration in livers of LPSinjected L-G9a ${ }^{-1-}$ males compared with the controls, which was absent in vehicle-injected groups (Fig. S3).

LPS induces liver injury mainly through inflammation. After LPS injection, increased F4/80 (marker for macrophages), Ly-6G (marker for neutrophils), and CD3 (marker for $\mathrm{T}$ cells) staining were detected in livers of the control males, while further increased infiltration of these immune cells were found in L-G9a ${ }^{-/-}$males (Fig. 2a). Consistently, 


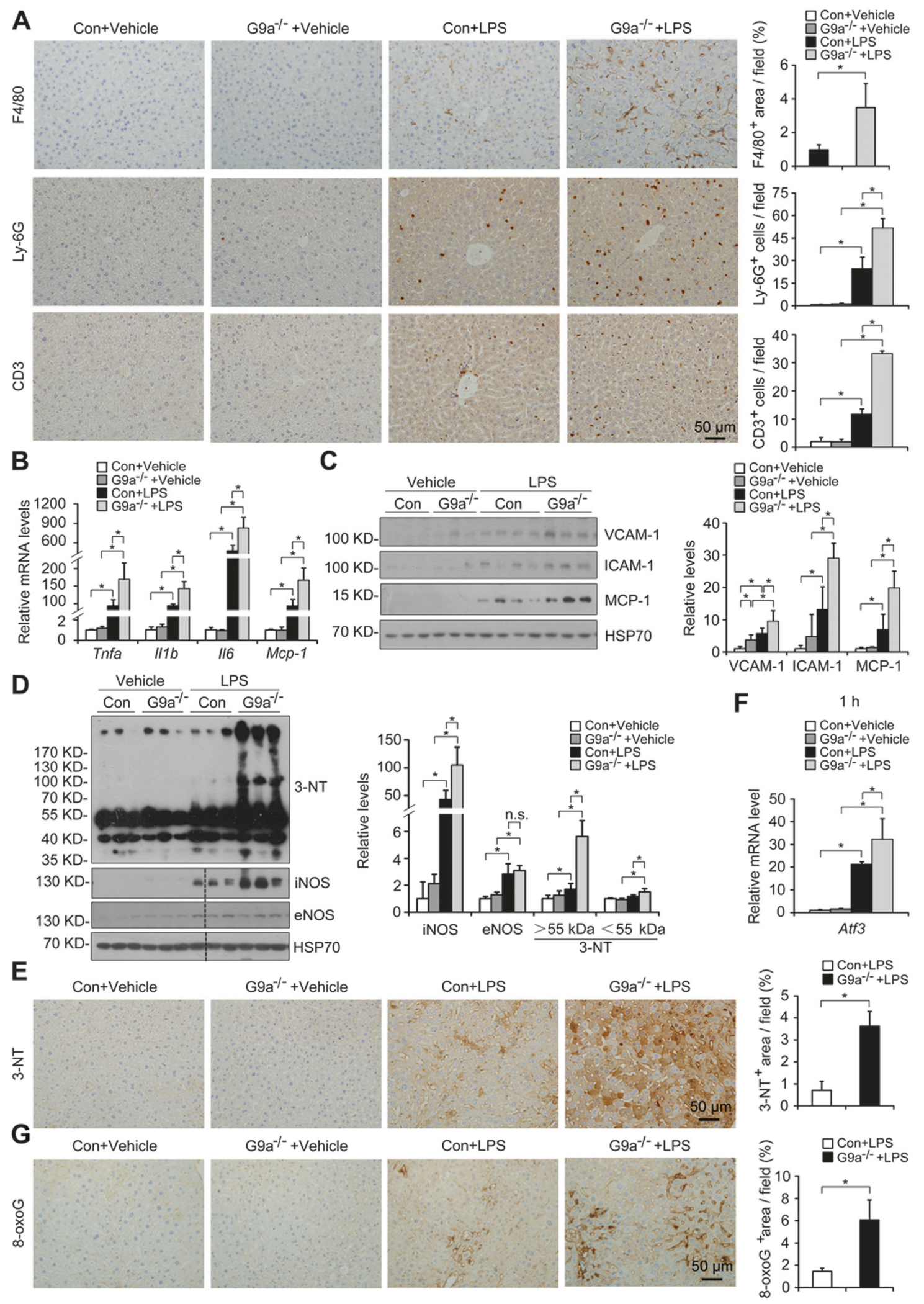

at $1 \mathrm{~h}$ after LPS injection, Tnfa, Illb, Il6, and monocyte chemotactic protein 1 (Mcp-1) were significantly elevated in the control livers, and further elevated in livers of L-G9 $\mathrm{a}^{-1-}$ males (Fig. 2b). Adhesion molecules, such as vascular cell adhesion molecule-1 (VCAM-1) and intercellular adhesion molecule-1 (ICAM-1), recruit leukocytes to injury sites to 
Fig. 2 Hepatic G9a deletion augments LPS-induced inflammation, RNS/ROS production, DNA damage and hepatocyte apoptosis. a Representative pictures for F4/80, Ly-6G and CD3 staining in the liver (left) and quantitative results (right). b qPCR results of the indicated genes in the liver of different groups. c, d Representative western blots (left) and quantitative results (right) for VCAM-1, ICAM-1, and MCP-1 (c), iNOS, eNOS and 3-NT (d) in livers of different groups. HSP70 serves as the loading control. e Representative pictures for 3-NT staining in the liver (left) and quantitative results (right). $\mathbf{f}$ Relative mRNA level of Atf 3 in the liver at $1 \mathrm{~h}$ after LPS injection. $g$ Representative pictures for 8-oxoG staining in the liver (left) and quantitative results (right). $n=3-4$ per group; 3-NT, 3Nitrotyrosine; n.s., not significant; ${ }^{*} p<0.05$; scale bar, $50 \mu \mathrm{m}$

initiate inflammatory responses. After LPS injection, increased levels of VCAM-1, ICAM-1, and MCP-1 were found in livers of the control males, while their levels were further elevated in L-G9a $\mathrm{a}^{-1-}$ males (Fig. 2c). Similar results were found in females (Fig. S4A-C).

RNS production augments inflammation and tissue injury [16]. After LPS injection, increased iNOS and eNOS levels were detected in livers of male controls, and further dramatically increased iNOS but not eNOS level was detected in L-G9a ${ }^{-1-}$ males (Fig. 2d). 3-nitrotyrosine (3NT) is a "molecular fingerprint" indicating the formation of nitrated proteins [17]. After LPS injection, significantly elevated nitrated proteins were found in livers of $\mathrm{L}-\mathrm{G} 9 \mathrm{a}^{-1-}$ males compared with the controls, despite of the similar levels of NOSs and nitrated proteins in these two groups under physiological conditions (Fig. 2d, e).

To test whether oxidative damage is involved in the exacerbated LPS-induced liver injury of L-G9a $\mathrm{a}^{-1-}$ mice, we measured the levels of Atf3 and 8-oxoguanine (8-oxoG), a ROS-induced transcription factor and a marker for oxidative damage, respectively [4]. Without LPS injection, no difference was found in livers of male L-G9a $\mathrm{a}^{-1-}$ and their controls (Fig. 2f, g). In livers of LPS-injected male controls, elevated Atf3 and 8-oxoG staining were found; which were further elevated in LPS-injected L-G9a ${ }^{-/-}$males (Fig. 2f, g). Similar results were seen in females (Fig. S4D-G).

\section{Hepatic G9a deletion augments DNA damage and hepatocyte apoptosis upon LPS injection}

Mounting evidences suggest that nitrative and oxidative stresses induce DNA damage [18]. After LPS challenge, the levels of p-Chk2, p-Chk1, p-p53 and p-H2A.X were dramatically increased in the controls, and were further increased in livers of L-G9a ${ }^{-1-}$ males; whereas the p53 level was similar in these groups (Fig. 3a). Hepatocyte apoptosis plays a pathogenic role in acute liver injury [18]. Without LPS challenge, the levels of apoptosis-associated proteins and the number of $\mathrm{TUNEL}^{+}$cells were similar in livers of $\mathrm{L}-\mathrm{G} 9 \mathrm{a}^{-1-}$ males and their controls (Fig. 3b, c).
After LPS injection, increased levels of apoptosis-related cleaved fragment of poly(ADP-ribose) polymerase-1 (PARP-1), cleaved Caspase3/8, and increased number of $\mathrm{TUNEL}^{+}$cells were found in livers of the controls; and these markers were further upregulated in livers of LPSinjected L-G9a $\mathrm{a}^{-1-}$ males (Fig. 3b, c). Similar results were found in females (Fig. S5A-C).

\section{Inhibiting G9a methyltransferase activity exacerbates LPS-induced liver damage}

To investigate whether G9a-deficiency enhanced acute liver damage depends on its methyltransferase activity, BIX01294 (BIX), an inhibitor for the methyltransferase activity of G9a, was used [9]. The specificity of BIX was first evaluated. In WT primary hepatocytes and HepG2 cells, BIX significantly downregulated H3K9me1/me2 levels (Fig. S6A-B). In $\mathrm{G}^{-1-} \mathrm{a}^{-1}$ primary hepatocytes, downregulated $\mathrm{H} 3 \mathrm{~K} 9 \mathrm{me} 1 / \mathrm{me} 2$ levels were found as expected, however, no further downregulation of H3K9me1/me2 levels was observed after BIX treatment (Fig. S6C). Consistently, BIX could not further downregulate hepatic $\mathrm{H} 3 \mathrm{~K} 9 \mathrm{me} 1 / \mathrm{me} 2$ levels in $\mathrm{L}-\mathrm{G} 9 \mathrm{a}^{-/-}$males (Fig. S6D). H\&E and F4/80 staining suggested BIX per se did not cause significant pathological changes; in contrast, BIX markedly increased accumulation of macrophages in the liver upon LPS injection (Fig. 4a, b). Consistently, inflammatory molecules such as VCAM-1, ICAM-1, MCP1, and iNOS, DNA damage marker p-H2A.X, as well as apoptosis markers like cleaved PARP-1 and Caspase3, were significantly increased in livers of BIX and LPS co-treated males, compared with those treated with either one (Fig. 4c, d). Moreover, compared with LPS-treated males, further elevated hepatic Atf3 was detected in co-treated males (Fig. 4e). These results suggest a methyltransferase activity dependent role of G9a on liver injury.

\section{Deletion of G9a exacerbates APAP-induced acute liver injury}

To further verify the role of G9a on acute liver injury, an APAP overdose model was used. At $24 \mathrm{~h}$ after a sublethal APAP overdose, L-G9a ${ }^{-1-}$ males displayed deteriorated physical states compared with the controls (Movie S1). In the controls, APAP overdose caused massive hepatic toxicity as indicated by gross injured liver (Fig. 5a), typical centrilobular necrosis demonstrated by H\&E staining (Fig. 5b), and increased serum ALT/AST levels (Fig. 5c). Under APAP stress, L-G9a ${ }^{-1-}$ males displayed more severe liver necrosis and much higher serum ALT/AST (Fig. 5a-c). The toxicity of APAP comes from its metabolite $\mathrm{N}$-acetyl benzoquinoneimine, which triggers hepatic damage by depleting glutathione (GSH) [19]. APAP 

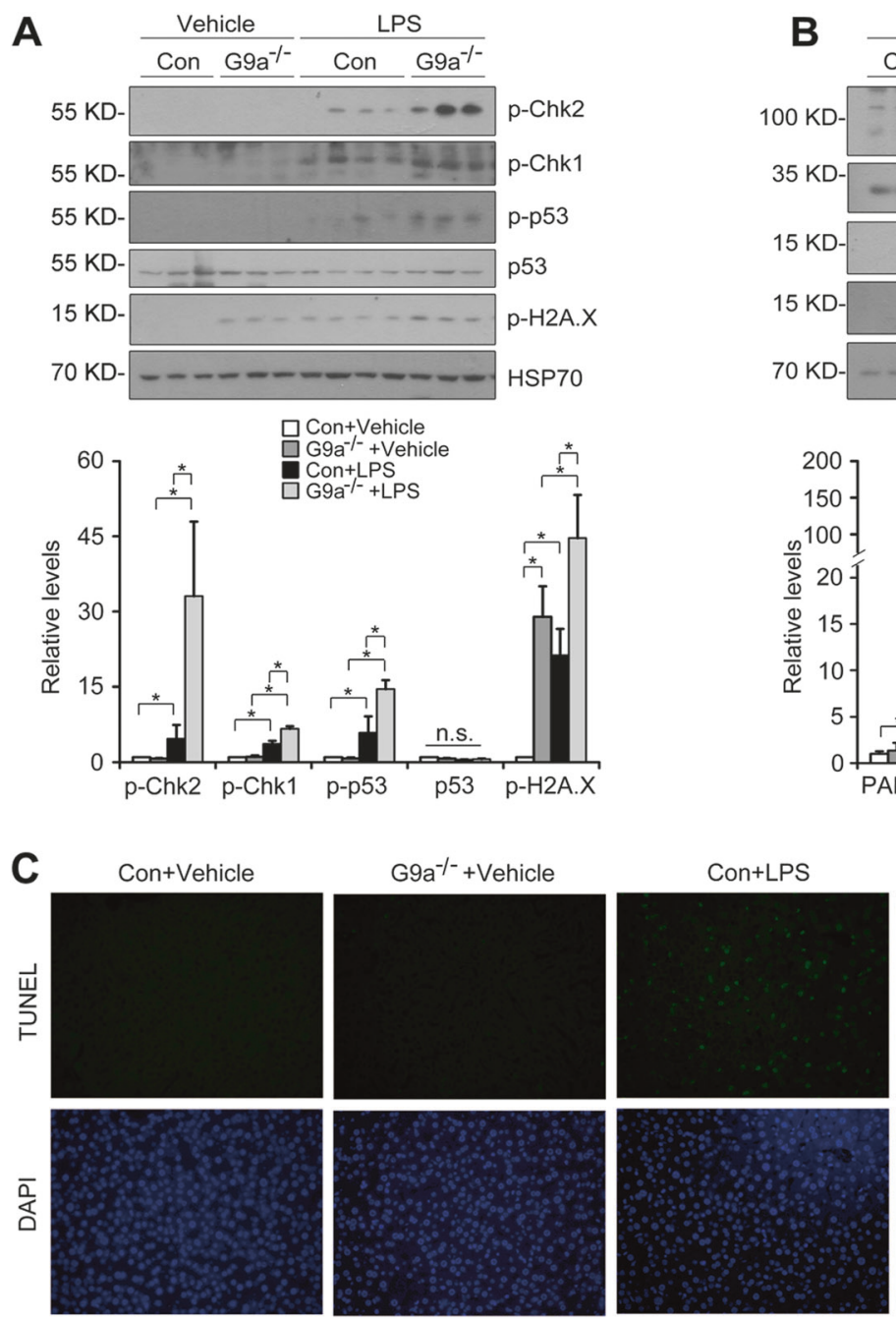

Fig. 3 Hepatic G9a deletion augments DNA damage and hepatocyte apoptosis upon LPS injection. a, b Representative western blots (up) and quantitative results (down) for markers of DNA damage response signaling (a) and apoptosis (b) in livers of different groups.

overdose significantly decreased hepatic GSH level of the control males, while more pronounced decrease was observed in L-G9a ${ }^{-1-}$ males (Fig. 5d), as well as aggravated oxidative stress indicated by elevated liver Atf3 and

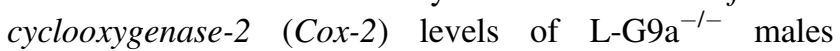
(Fig. 5e). APAP overdose also dramatically upregulated the hepatic iNOS and 3-NT in livers of L-G9 $\mathrm{a}^{-l-}$ males compared with the controls (Fig. 5f). Meanwhile, after APAP overdose, increased Ly-6G staining was detected in the control livers, while further increased neutrophils infiltration was found in $\mathrm{L}-\mathrm{G} 9 \mathrm{a}^{-1-}$ mice (Fig. $5 \mathrm{~g}$ ).

\section{Loss of G9a represses Gstp1/2}

Compared with the controls, Coomassie brilliant blue staining of whole liver lysate indicated a faint band in L-G9a ${ }^{-1-}$ males about $23 \mathrm{kDa}$ with or without LPS challenge
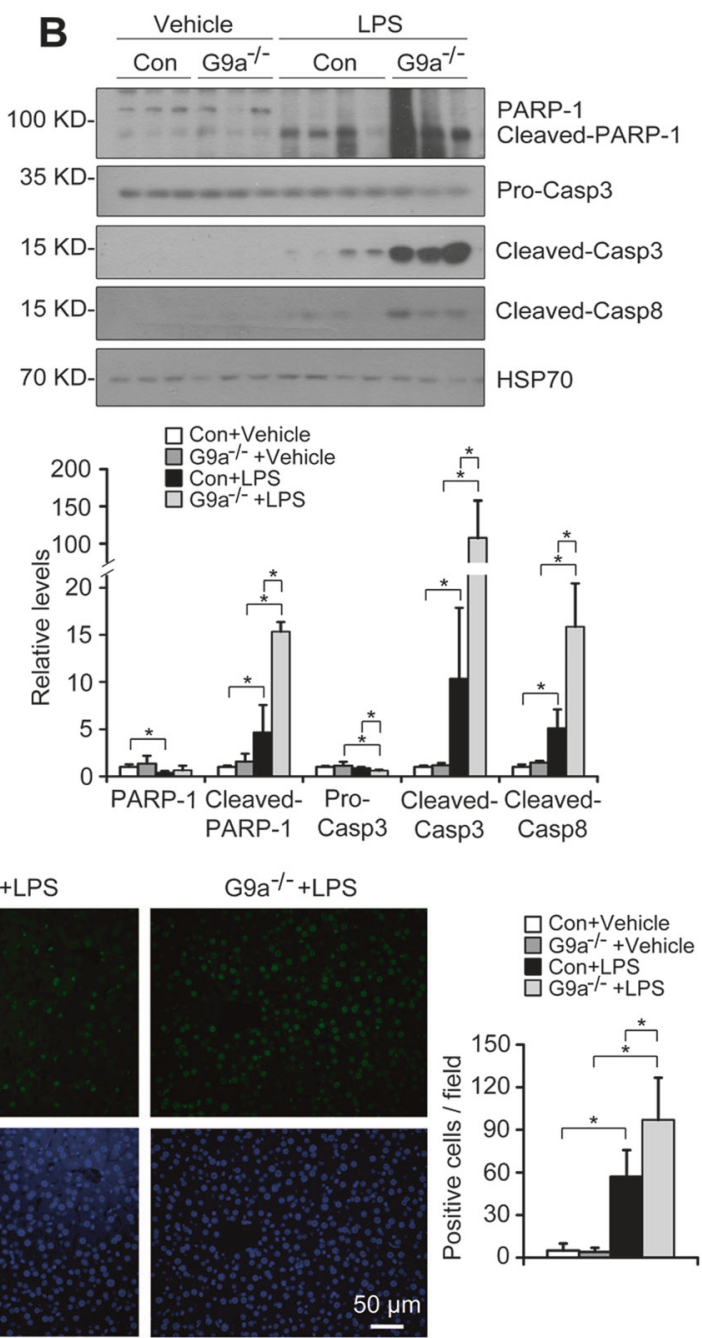

HSP70 serves as the loading control. c Representative pictures for TUNEL staining in the liver (left) and quantitative results (right). All data were obtained from male mice. $n=3-4$ per group; n.s., not significant; ${ }^{*} p<0.05$; scale bar, $50 \mu \mathrm{m}$

(Figs. 6a, S7), further MS study identified two top candidates, GSTP1 and GSTP2 (Fig. S8). GSTP is an isoform of glutathione S-transferases (GSTs), which are a family of phase II detoxifying enzymes. Mouse has two GSTP genes, Gstp1 and Gstp2 [20]. We found Gstpl is much more abundant than Gstp2 in mouse liver (Fig. S9A). The levels of GSTP $1 / 2$ were significantly decreased in livers of L-G9a ${ }^{-/-}$ mice compared with the controls, with or without LPS challenge; and LPS dramatically downregulated GSTP1/2 levels in the controls (Figs. 6b, c, S9B). Consistently, in a recent study, genes of six different pathways were screened by real-time PCR in L-G9a ${ }^{-1-}$ mice, and GSTP1 is one of the more than 80 altered genes screened with a real-time PCR approach [21]. Furthermore, BIX treatment decreased GSTP1/2 levels with or without LPS stress (Fig. S9C), indicating a G9a methyltransferase activity dependent regulation of GSTP1/2. Consistently, APAP overdose 

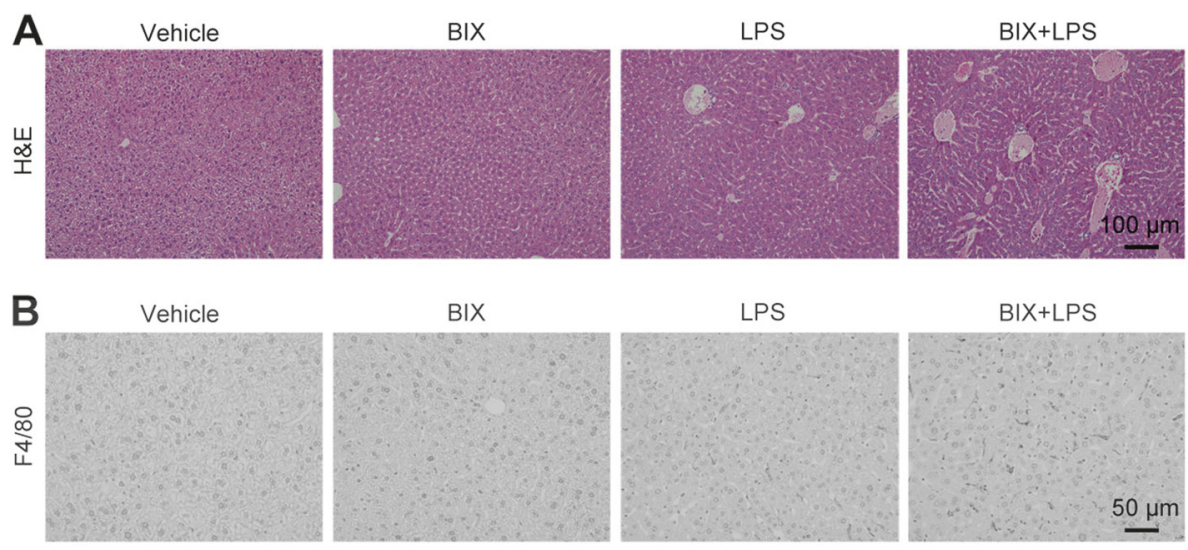

$\mathrm{BIX}$

LPS

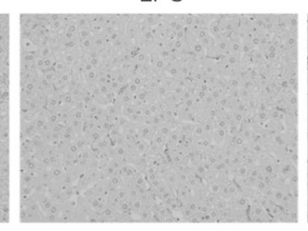

$B \mid X+L P S$
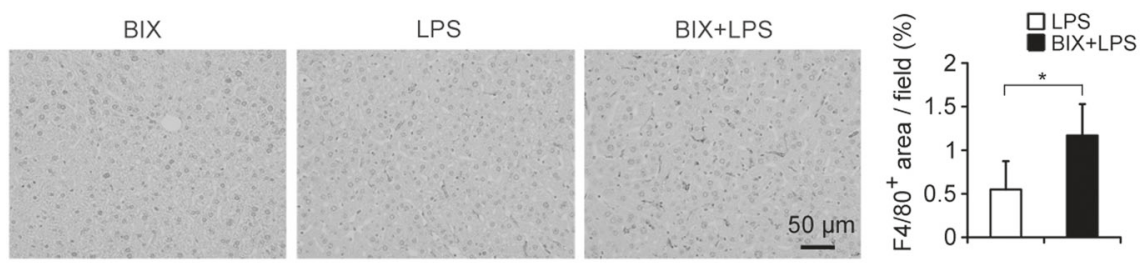
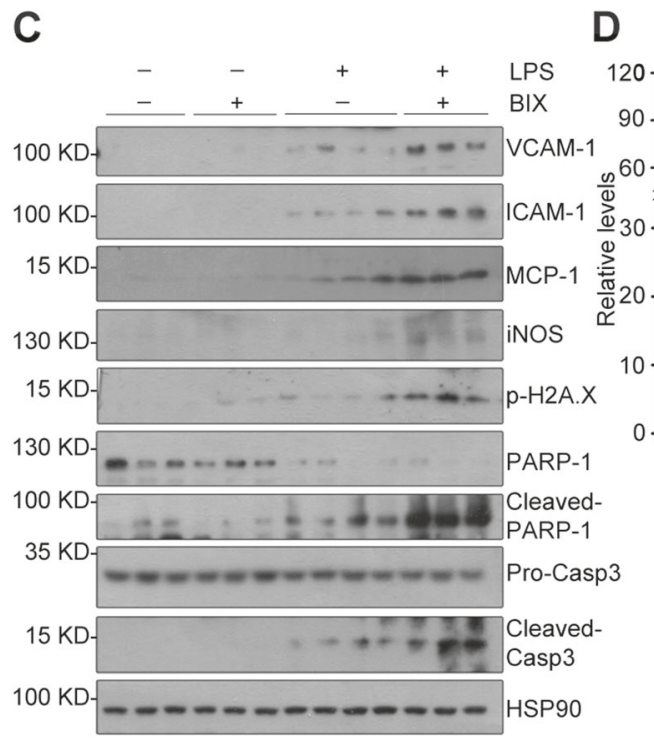

Fig. 4 Inhibiting G9a methyltransferase activity deteriorates LPSinduced liver damage. a, b Representative pictures for H\&E staining (a) scale bar, $100 \mu \mathrm{m}$ ); and F4/80 staining (b, left; scale bar, $50 \mu \mathrm{m}$ ) with quantitative results (b, right) in the liver. c, d Representative

downregulated GSTP1/2 levels moderately in the controls and drastically in L-G9a ${ }^{-1-}$ mice (Fig. 6d). Since GSTPs not only play roles in protecting cells against toxins (drugs and carcinogens) and oxidative stress, but also have antiinflammation and anti-apoptosis effects [20, 22], we thus hypothesize that the downregulation of GSTP1/2 in livers of L-G9a $\mathrm{a}^{-/-}$mice contributes to the exacerbated liver injury upon LPS or APAP overdose stress.

\section{G9a mediates H3K9me1 at the promoters of Gstp1/2}

We next investigated the mechanisms by which G9a regulates Gstp1/2. Transcription factor Nrf2 (NFE-2 related factor 2) regulates expression of GSTPs [23]. Under normal condition, Nrf2 binds to Keap1 (Kelch-like ECH-associated protein 1) in cytoplasm. Upon oxidative stress, the Nrf2-Keap1 complex is disrupted, the released

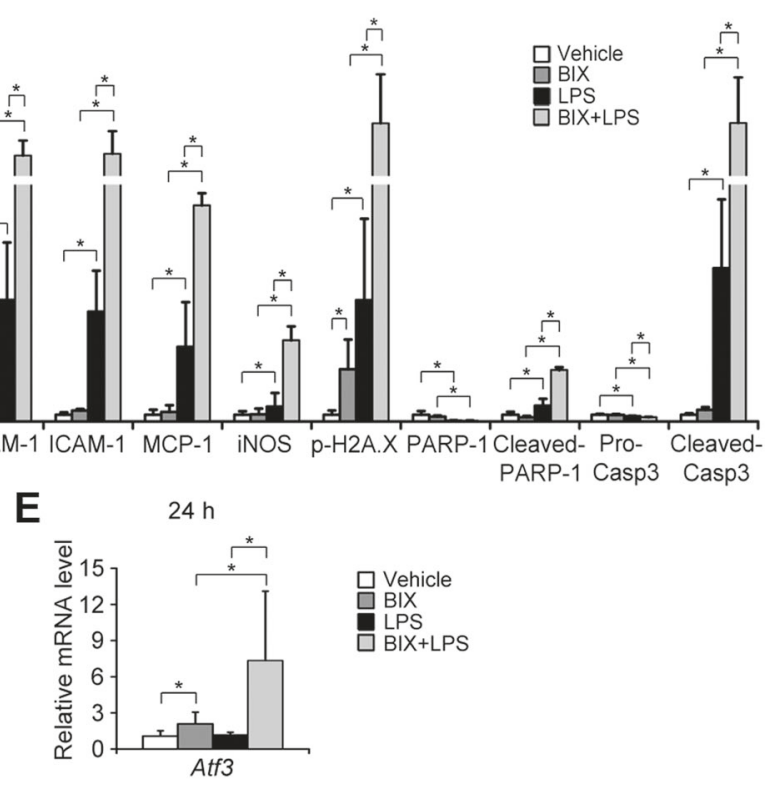

western blots (c) and quantitative results (d) of indicated proteins in livers of different groups. HSP90 serves as the loading control. e Relative mRNA level of Atf 3 in the liver at $24 \mathrm{~h}$ after LPS injection. $n=3-4$ per group; ${ }^{*} p<0.05$

Nrf2 translocates to the nucleus and activates targeted genes [24]. We tested whether G9a deficiency inhibits Gstp1/2 expression via repressing the Keap1-Nrf2 axis. We found no differences on the total levels of Nrf2 and Keap1 in livers of the controls and L-G9a ${ }^{-/-}$males with or without LPS injection; however, LPS did induce significant downregulation of total Nrf2 and Keap1 levels in these two groups (Fig. S9D, E). Furthermore, similar nuclear Nrf2 staining were observed in livers of the controls and L-G9a $\mathrm{a}^{-1-}$ males; whereas LPS-treatment induced similar reduction of total and nuclear $\mathrm{Nrf} 2$ in livers of both groups (Fig. S9F). Consistently, the levels of multiple Nrf2 targeted genes, including Hmox1, Nqo1, $\mathrm{Gcl}$, and Mrpl, were similar in livers of the control and L-G9a ${ }^{-1-}$ mice, with or without LPS injection (Fig. S9G). Thus, the alteration of GSTP1 in L-G9a ${ }^{-1-}$ mice may not due to changes in Nrf2 signaling. 

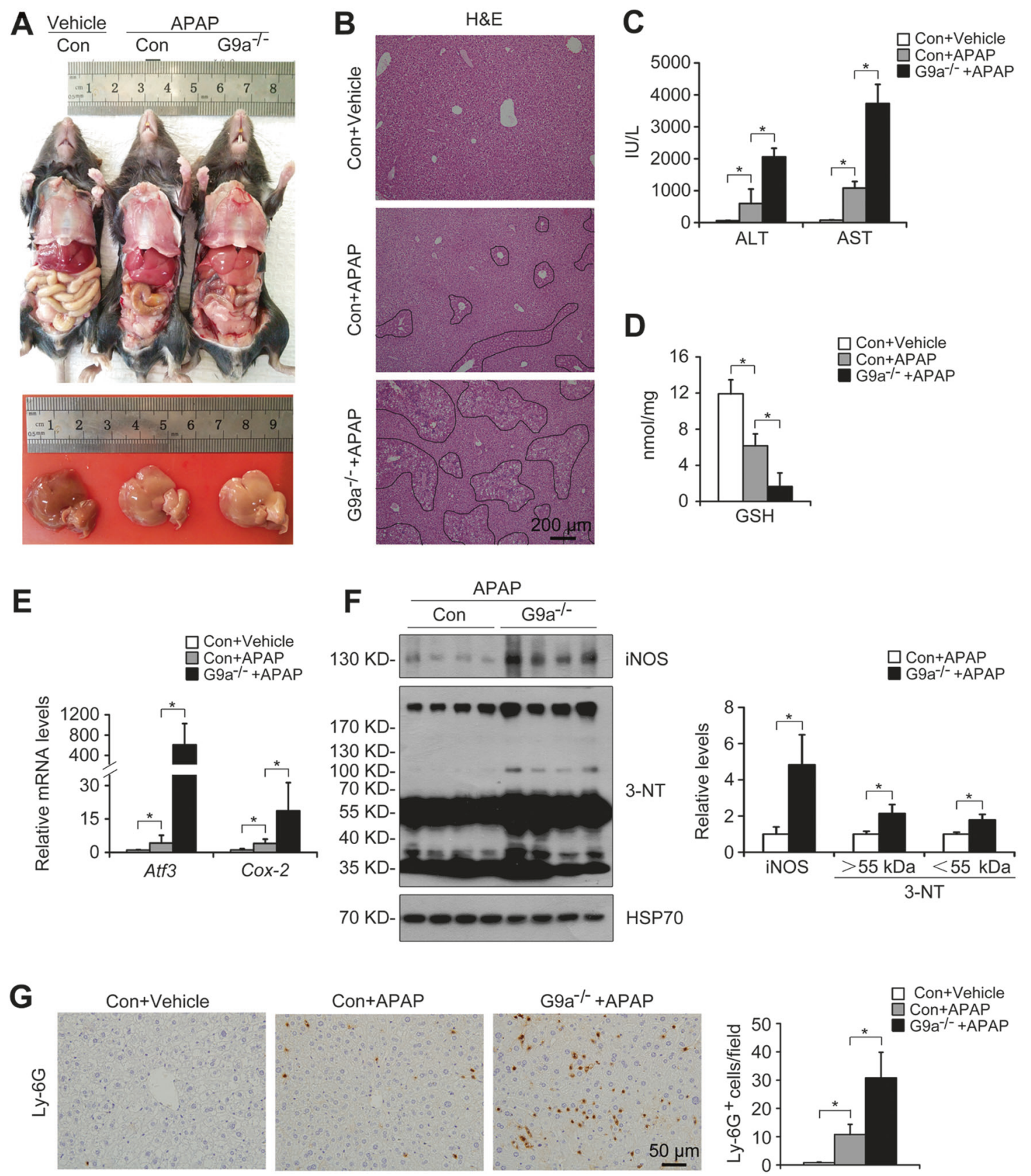

Fig. 5 Deletion of G9a exacerbates APAP overdose-induced acute liver injury. a Representative pictures of livers from different groups at $24 \mathrm{~h}$ after APAP injection. b Representative pictures for H\&E staining in the liver; scale bar, $200 \mu \mathrm{m}$. c Serum levels of ALT and AST. d Levels of GSH in the liver. e Relative mRNA levels of Atf3 and Cox-

G9a regulates gene expression by adding repressive marker $\mathrm{H} 3 \mathrm{~K} 9 \mathrm{me} 2$ or transactivating marker $\mathrm{H} 3 \mathrm{~K} 9 \mathrm{me} 1$ at gene promoters [9], thus we hypothesize that G9a may directly regulate Gstpl/2 through epigenetic modification. ChIP assay indicated strong enrichment of H3K9me1, but no $\mathrm{H} 3 \mathrm{~K} 9 \mathrm{me} 2$, in the promoter regions $(-2000 \mathrm{bp}$ to TSS, transcription start site) of Gstpl/2 in livers of wildtype mice (Fig. S10). In the controls, the H3K9me1 levels were mildly

2 in the liver. f Representative western blots (left) and quantitative results (right) for the indicated proteins in the liver of different groups. HSP70 serves as the loading control. g Representative pictures for Ly6G staining in the liver (left) and quantitative results (right); scale bar, $50 \mu \mathrm{m} . n=4$ per group; ${ }^{*} p<0.05$

repressed in some promoter regions of Gstp1/2 upon LPS injection (Fig. 6e); whereas G9a-deficiency significantly reduced the H3K9me1 levels at the promoters of Gstp1/2 with or without LPS stress, and the H3K9me1 levels were further decreased in some promoter regions of Gstpl in livers of L-G9a ${ }^{-1-}$ mice upon LPS injection (Fig. 6e). In the controls, G9a bound to the same promoter regions of Gstpl, and G9a-deficiency significantly reduced binding (Fig. 6f). 


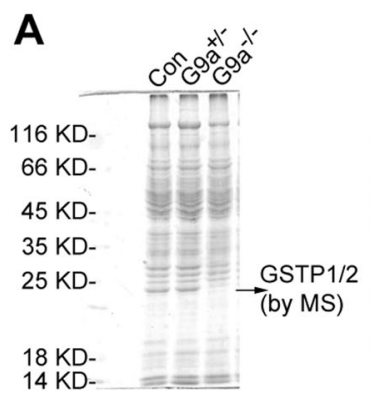

D

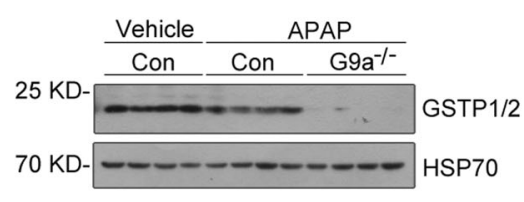

$\mathbf{F}$

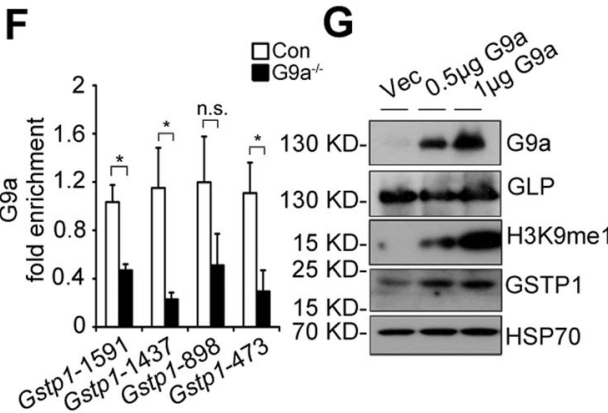

Fig. 6 G9a mediates H3K9me1 at the promoters of Gstp. a Coomassie brilliant blue staining of whole liver extracts from Control, L-G9a ${ }^{+/-}$ and L-G9a ${ }^{-1-}$ mice. b Relative mRNA levels of Gstp1 and Gstp2 in the liver of different groups at $24 \mathrm{~h}$ after LPS injection. c, d Representative western blots of GSTP1/2 (left) and quantitative results (right) in livers of different groups. HSP70 serves as the loading control. e ChIP-qPCR analysis of H3K9me1 levels at the promoters of Gstp1 and Gstp2 in livers of different groups at $24 \mathrm{~h}$ after LPS injection. f ChIP-qPCR analysis of G9a levels at the promoter of Gstp1

However, decreased $\mathrm{H} 3 \mathrm{~K} 9 \mathrm{me} 2$ levels at the promoters of inflammatory genes, such as Tnfa, $I l l b$, and $I l 6$, were seen in livers of L-G9a ${ }^{-1-}$ mice upon LPS injection (Fig. S11).

Next, we overexpressed G9a in wildtype primary hepatocytes. Increased levels of H3K9me1 and GSTP1/2, but not GLP, were observed (Fig. 6g). Re-constitution of G9a in L-G9a ${ }^{-1-}$ primary hepatocytes rescued downregulated H3K9me1/me2 and GSTP1/2 (Fig. 6h); meanwhile, ChIP assay demonstrated rescued $\mathrm{H} 3 \mathrm{~K} 9 \mathrm{me} 1$ binding to the promoter of Gstpl (Fig. 6i).

\section{rGSTP1 inhibits the aggravated liver injury induced by LPS or APAP overdose in G9a-dificient mice}

To address whether the protective effects of G9a on LPS- or APAP-induced liver damage is mediated by GSTPs, we
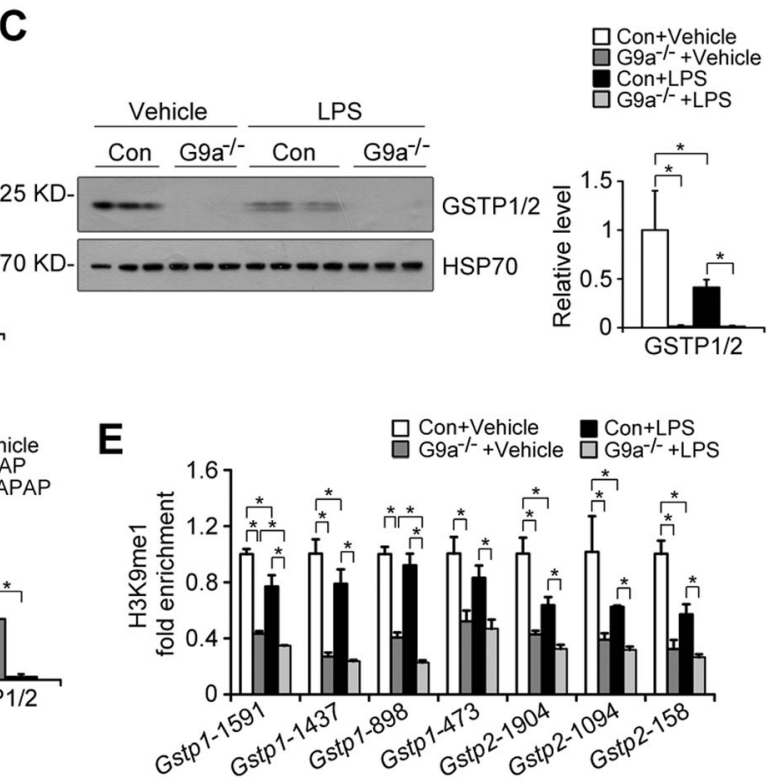

H

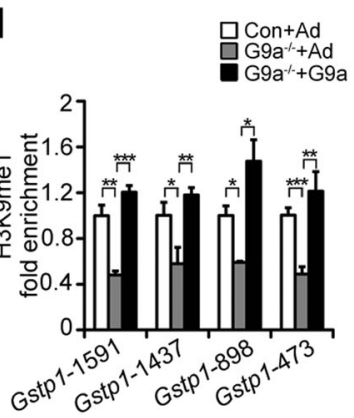

in livers of control and L-G9a ${ }^{-1-}$ mice (error bars indicate SEM). Above data (a-f) were obtained from mice with $n=3-4$ per group. $\mathbf{g}$, h Representative western blots of GSTP1/2, G9a, H3K9me1, H3K9me2, or GLP after overexpression of pCAGGS-mG9a in primary hepatocytes obtained from WT (g) or G9a ${ }^{-l-}(\mathbf{h})$. HSP70 serves as the loading control. Similar results were observed from the Ad-G9a transfection. i ChIP-qPCR analysis of H3K9me1 levels at the promoters of Gstpl in control, G9a ${ }^{-1-}$ or adenovirus-mediated G9a transfected $\mathrm{G} \mathrm{a}^{-1-}$ primary hepatocytes. ${ }^{*} p<0.05$

administered rGSTP1 to L-G9a ${ }^{-/-}$mice. Exogenous rGSTP1 effectively rescued the LPS-induced GSTP1/2 reduction in the livers without significantly affecting H3K9me1/me2 levels (Fig. 7a). Under LPS injection, rGSTP1-treated mice showed better physical activity (Movie S2), as well as significantly lower serum ALT/AST levels (Fig. 7b). rGSTP1 also significantly inhibited LPSinduced accumulation of macrophages and nitrated proteins, elevation of VCAM-1, MCP-1, iNOS, and 3-NT levels, and upregulated some antioxidant genes such as Sod2, Cat, $\operatorname{Tr} x 1$, and Gpx2, in livers of $\mathrm{L}-\mathrm{G} 9 \mathrm{a}^{-1-}$ mice (Fig. 7c-e, S12A). Furthermore, rGSTP1 significantly inhibited DNA damage and hepatocyte apoptosis induced by LPS in livers of L-G9a ${ }^{-1-}$ mice (Fig. 7f).

Similarly, at $24 \mathrm{~h}$ post APAP overdose, rGSTP1-treated L-G9a ${ }^{-/-}$mice also showed better behavior and physical 
A
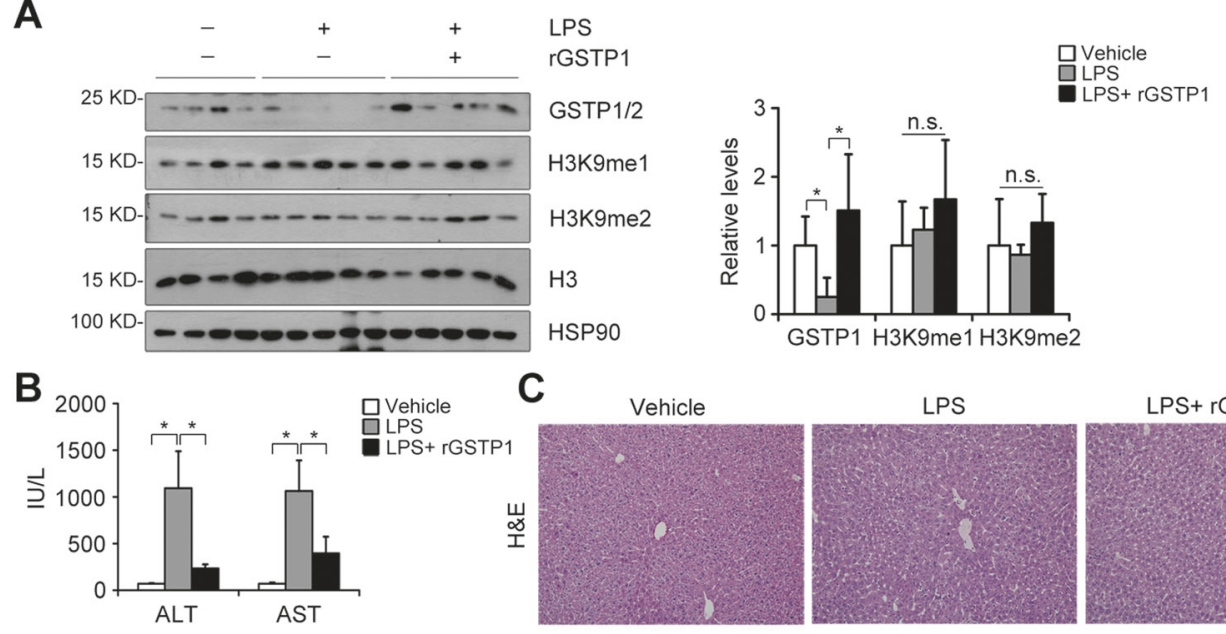

C

Vehicle

LPS

LPS+ rGSTP1
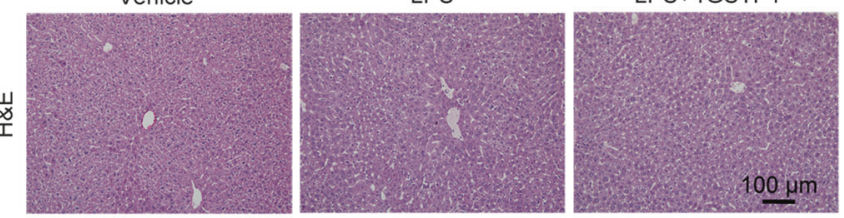

D

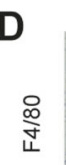

Vehicle

LPS

LPS+ rGSTP1
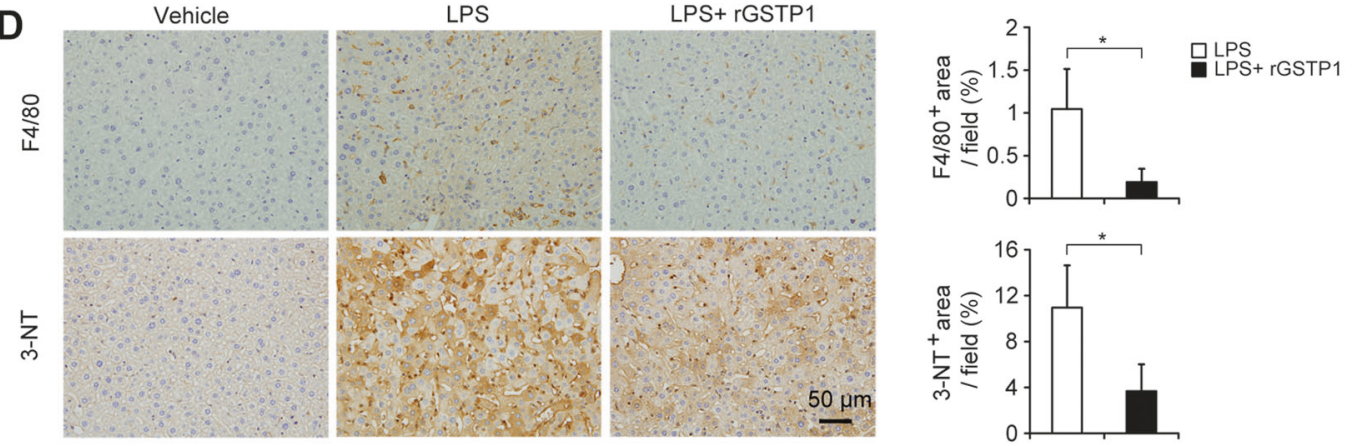

E

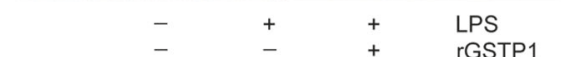

F
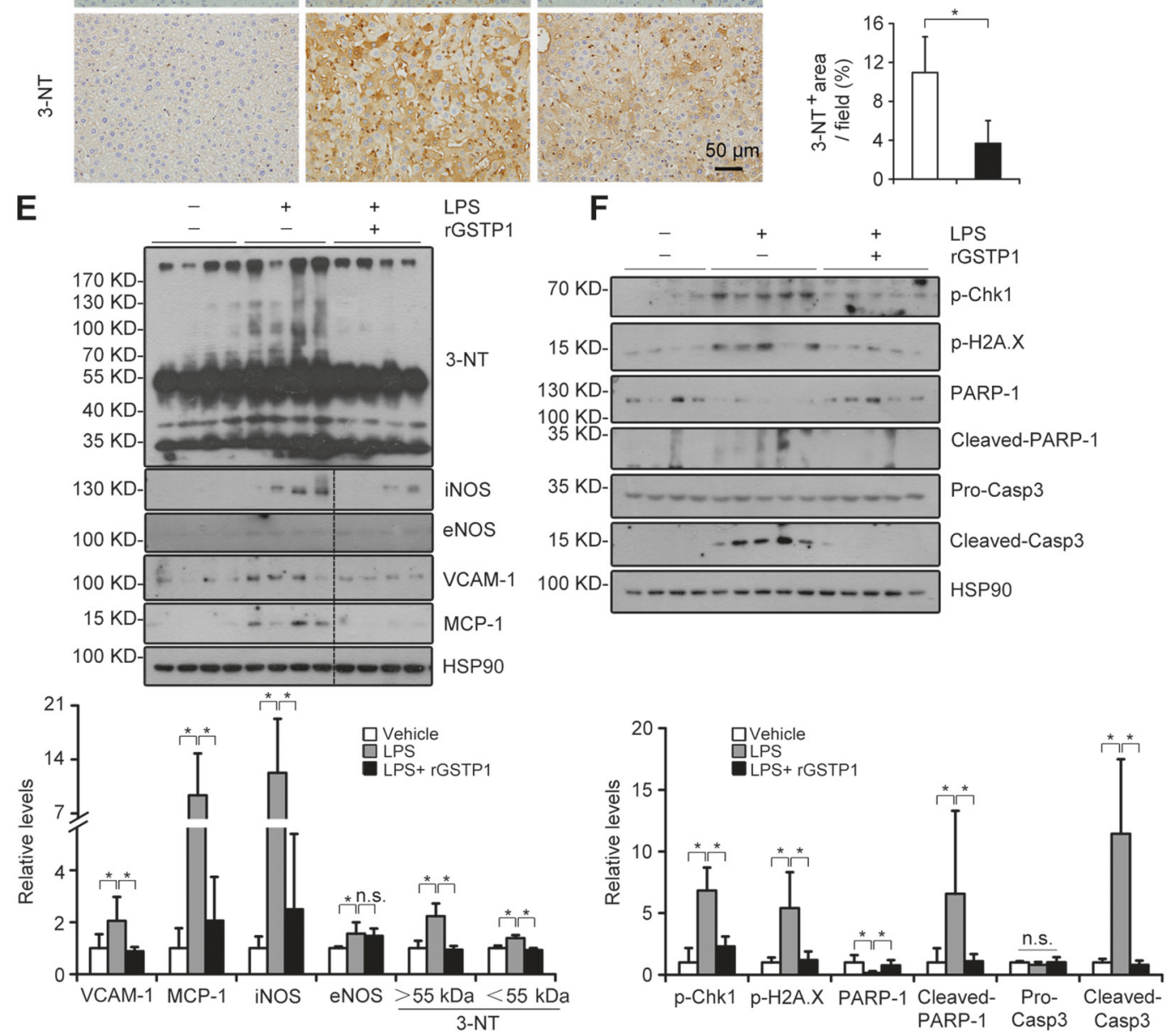

Fig. 7 rGSTP1 attenuates LPS-induced liver damage in L-G9a ${ }^{-1-}$ mice. a Representative western blots (left) and quantitative results (right) for GSTP1/2, H3K9me1/me2 in the livers of different groups. H3 or HSP90 serves as the loading control. b Serum levels of ALT and AST. c Representative pictures for H\&E staining in the liver; scale bar, $100 \mu \mathrm{m}$.

d Representative pictures for F4/80 and 3-NT staining (left) and quantitative results (right); scale bar, $50 \mu \mathrm{m}$. e, f Representative western blots (up) and quantitative results (down) of the indicated proteins in livers of different groups. HSP90 serves as the loading control. All these data were obtained from male L-G9a ${ }^{-1-}$ mice. $n=4-6$ per group; ${ }^{*} p<0.05$ 
activity (Movie S3), alleviated hepatic toxicity (Fig. 8a) and centrilobular necrosis (Fig. 8b), as well as lower serum ALT/AST levels (Fig. 8c). Moreover, as an important detoxifying protein, rGSTP1 prevented $\mathrm{L}-\mathrm{G} 9 \mathrm{a}^{-/-}$mice from GSH depletion (Fig. 8d), and oxidative damages as indicated by the lower Atf3 level (Fig. 8e), as well as potentiated some antioxidant genes (Fig. S12B). Furthermore, rGSTP1 also significantly inhibited APAP-induced nitrated proteins accumulation in livers of $\mathrm{L}-\mathrm{G} \mathrm{a}^{-1-}$ mice (Fig. 8f). Ly-6G staining suggested significantly decreased neutrophils infiltration in livers of rGSTP1-injected
L-G9a ${ }^{-1-}$ mice compared with the controls (Fig. 8g). Together, these results demonstrate that rGSTP1 protects LPS- or APAP overdose-induced acute liver injury in $\mathrm{L}-\mathrm{G} 9 \mathrm{a}^{-/-}$mice.

Whether rGSTP1 can be transported into cells was examined. FITC-labeled rGSTP1, but not free FITC, could be uptaken by Hepa1-6 cells (Fig. S13A), which was consistent with a previous report that rGSTP1 can be transported into cardiac tissue [22]. Moreover, transported rGSTP1 was markedly inhibited by CPZ or dynasore, two inhibitors for the clathrin-dependent endocytosis, but not by
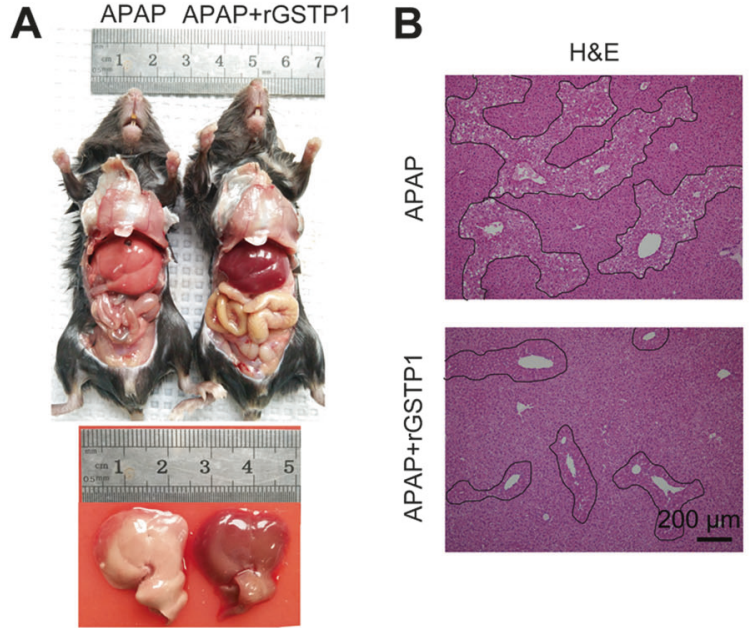

$\mathbf{F}$
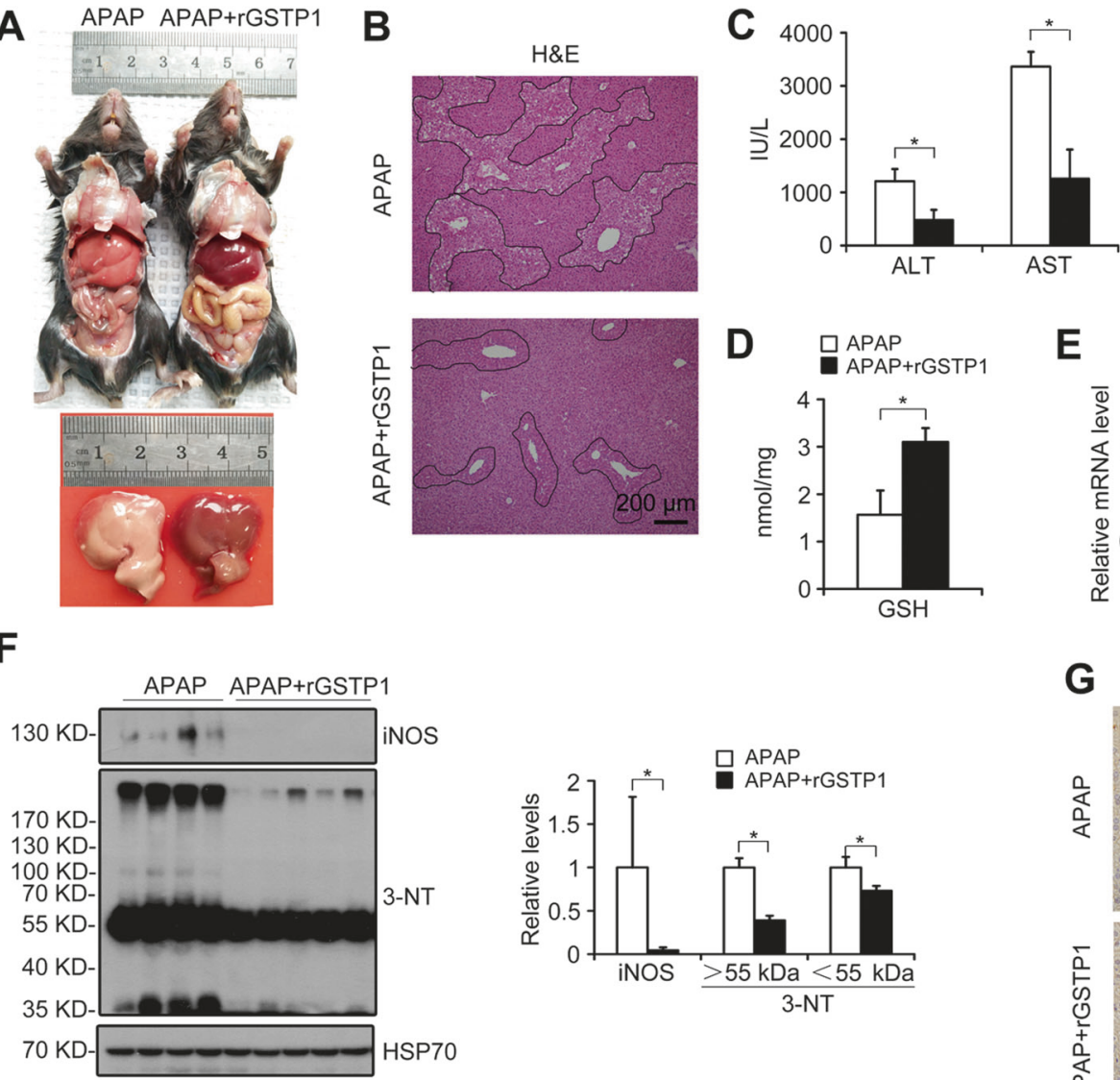

E

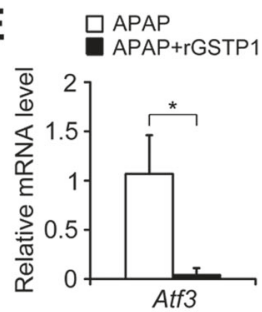

G
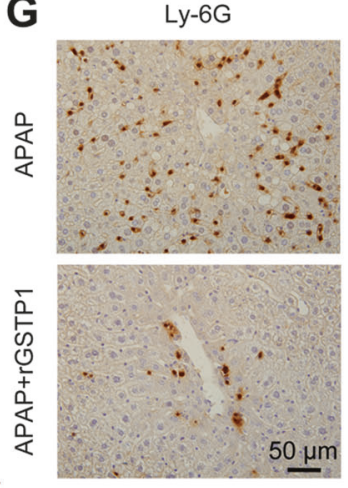

H
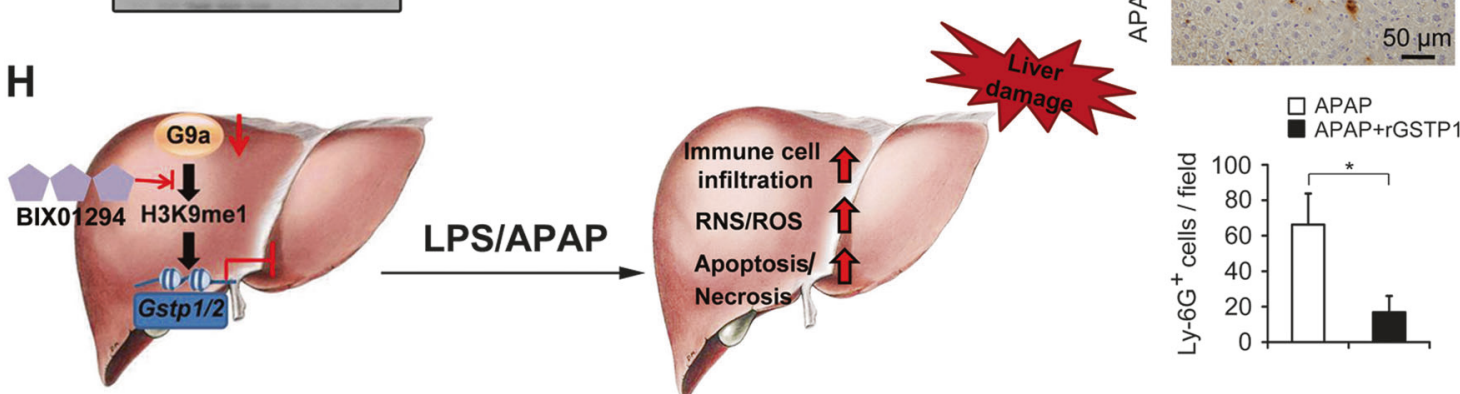

Fig. 8 rGSTP1 attenuates APAP-induced liver damage in L-G9a ${ }^{-1-}$ mice. a Representative pictures of livers from different groups at $24 \mathrm{~h}$ after APAP injection. b Representative pictures for H\&E staining in the liver; scale bar, $200 \mu \mathrm{m}$. c Serum levels of ALT and AST. d Levels of GSH in the liver. e Relative mRNA level of Atf 3 in the liver. f Representative western blots (left) and quantitative results (right) for the indicated proteins in livers of different groups. HSP70 serves as the loading control. g Representative pictures for Ly-6G staining in the liver (up) and quantitative results (down); scale bar, $50 \mu \mathrm{m}$. All these data were obtained from male L-G9a ${ }^{-1-}$ mice. $n=4-6$ per group; n.s., not significant; ${ }^{*} p<0.05$. h Working model for how G9a regulates acute liver injury 
$\mathrm{M} \beta \mathrm{CD}$, an inhibitor for the caveolae-dependent endocytosis (Fig. S13B).

\section{Discussion}

Acute liver injury, which has extremely poor prognosis and high mortality, lacks effective therapies. Here, we found liver-specific abrogation of G9a lead to aggravated acute liver injury upon LPS challenge in both genders. Mechanically, G9a upregulates GSTP1/2 levels, the liver detoxifying enzyme. Using two rodent models, we demonstrated that rGSTP1 treatment significantly rescued the acute liver injury in the L-G9a ${ }^{-1-}$ mice, based on liver function (indicated by ALT/AST levels), greater than $60 \%$ rescue effects were achieved by rGSTP1 treatment in both models at the dosage used (Figs. 7b, 8c).

$\mathrm{H} 3 \mathrm{~K} 9 \mathrm{me} 1$ and $\mathrm{H} 3 \mathrm{~K} 9 \mathrm{me} 2$ are generally regarded as epigenetic markers for gene activation and repression respectively, therefore altering G9a level may lead to changes of significant amount of genes. Consistently, previous reports have suggested that among differentially expressed genes, a portion (28-50\%) are positively regulated by G9a [9]. Interestingly, although G9a regulates both $\mathrm{H} 3 \mathrm{~K} 9 \mathrm{me} 1$ and $\mathrm{H} 3 \mathrm{~K} 9 \mathrm{me} 2$ levels, most studies have been focused on the role of $\mathrm{H} 3 \mathrm{~K} 9 \mathrm{me} 2$. Our results suggested that G9a regulates Gstpl through maintaining H3K9me1 levels at its promoter in the liver and cultured primary hepatocytes (Fig. 6e-i). It has been demonstrated that G9a positively regulates the transcription of $P H G D H$ and $P S A T 1$, two ratelimiting enzymes for de novo serine biosynthesis, through regulating $\mathrm{H} 3 \mathrm{~K} 9 \mathrm{me} 1$ levels [9]. Similarly, BIX also prevents renal fibrosis by downregulating klotho with decreased H3K9me1 [25].

Our results showed globally affected levels of $\mathrm{H} 3 \mathrm{~K} 9 \mathrm{me} 1$ and H3K9me2 by G9a-deficiency, but only $\mathrm{H} 3 \mathrm{~K} 9 \mathrm{me} 1$ seemed to be regulated on Gstpl promoter ( $-2000 \mathrm{bp}$ to TSS). This is not unusual for epigenetic regulations. In Drosophila, an enzyme activity impaired mutation of Trr, an $\mathrm{H} 3 \mathrm{~K} 4 \mathrm{me} 1$ methyltransferase, significantly altered H3K4me1 levels; however, H3K4me1 levels were unchanged in some genes [26]. Furthermore, ChIP-seq revealed significantly changed H3K9me1 but not H3K9me2 levels on PHGDH and SHMT2 gene loci after manipulating G9a level [9]; compared with the amount of H3K9me1 binding, H3K9me2 binding on SHMT2 gene loci are much less [9]. Another report showed H3K9me2 but not H3K9me1 enrichment on the promoter or 5'-upstream region of some target genes of G9a, such as Mege-a2 or Wfdc15a [27]. However, questions like how many types of modifications are simultaneously involved in regulating a specific gene promoter region, and the exact roles these specific enrichments in combination play, remain unclear.
The role of $\mathrm{H} 3 \mathrm{~K} 9 \mathrm{me} 2$ on gene regulation may also contribute to protective effects of G9a on acute liver injury. Ablation or pharmacological suppression of G9a inhibits H3K9me2 on the promoter of Beclin-1, triggers the accumulation of intracellular ROS and activates NF- $\kappa \mathrm{B}$ pathway in MCF-7 cells [28]. Furthermore, G9a methyltransferasedependent regulation of $\mathrm{H} 3 \mathrm{~K} 9 \mathrm{me} 2$ on the promoter of inflammatory genes, such as TNF $\alpha$ in THP-1 monocyte and $I F N-\alpha / \beta$ in MEFs, has been reported [29, 30]. Consistently, here we reported in vivo, that genetically or pharmacologically inhibiting G9a caused aggravated inflammation and ROS production in acute liver injury, which may in part due to the repression of $\mathrm{H} 3 \mathrm{~K} 9 \mathrm{me} 2$ enrichment on the promoters of Tnfa, Illb, and Il6 (Figs. 2, 4, 5e-g, S4 and S11).

Besides function as a histone methyltransferase, G9a also can act in a histone methylation-independent but enzymatic activity dependent manner. By methylating estrogen receptor $\alpha$, G9a can work as co-activator of nuclear receptors [31]. Whether G9a also works independent of histone methylation in acute liver injury awaits further investigation.

Notably, rGSTP1 treatment significantly inhibited LPSor APAP-induced immune cells infiltration, RNS/ROS production, hepatocyte death and liver injury in L-G9a ${ }^{-/-}$ mice (Figs. 7, 8), suggesting that the aggravated liver damage induced by G9a deficiency was mediated, at least in part, by rGSTP1. Previous studies have shown that extracellular GSTP1 can cross plasma membrane, and rGSTP1 protects against infarction-induced heart failure and LPSinduced acute lung injury by reducing infarct area, apoptosis, and inflammation [22, 32]. Here, we also demonstrated that rGSTP1 can be transported into cells, possibly via clathrin-dependent endocytosis (Fig. S13). Despite of drastically reduced GSTP1/2 levels in liver, $\mathrm{L}-\mathrm{G} 9 \mathrm{a}^{-/-}$mice exhibited no obvious physical defect under normal conditions; consistently, pathological investigation on liver reveals no significant abnormality [33]. However, $\mathrm{GSTP}^{-1-}$ mice are more susceptible to stress-induced organ injuries, including urinary bladder, skin and heart, exhibiting more aggravated inflammation, oxidative stress, apoptosis and consequently higher mortality [34-36]. GSTP1 catalyzes Sglutathionylation of a variety of highly genotoxic and celldamaging molecules, and facilitates a variety of redox genes expression. Here, we found dramatically increased glutathione level, upregulation of several antioxidant genes, and decreased levels of iNOS and 3-NT, after rGSTP1 treatment (Fig. 8d-f, S12), suggesting the detoxification and redox function of rGSTP1 contribute to its hepatic protective effects.

In summary, our results indicate that G9a is essential in protecting against acute liver injury (Fig. 8h). Liver specific G9a-deficient mice show greater immune cells infiltration, RNS/ROS production and cell death upon endotoxin or APAP stimulation. G9a-regulated H3K9me1 at the 
promoters of Gstp 1/2 is responsible for the pathological and biochemical changes in endotoxin- or APAP-injected $\mathrm{L}-\mathrm{G} 9 \mathrm{a}^{-1-}$ mice, and rGSTP1 is necessary and sufficient to attenuate liver injury in $\mathrm{L}-\mathrm{G} 9 \mathrm{a}^{-1-}$ mice. Our study thus uncovers an epigenetic regulatory role of G9a in acute liver injury, and provides a molecular explanation for the beneficial role of G9a-GSTP1 axis against such injuries.

Acknowledgements The work is technically supported by the Analytical and Testing Core of College of Life Sciences of Wuhan University, and by the Analytical and Testing Center of HUST. This work is supported by the National Key $R \& D$ Program of China (2018YFA0800700), the Natural Science Foundation of China (31671195, 31871381, 31971066 \& 31871411), the Front Youth Program of HUST, and the Natural Science Foundation of Hubei Province (2016CFA012).

Author contributions KH, LZ, WX, YZ, SW designed research; YZ, WX, WZ, YY, XZ, QW, YW, DY, CY, YC, YS performed experiments; YZ, WX, XZ, KH, LZ analyzed data; WX, YZ, KH, LZ wrote the manuscript.

\section{Compliance with ethical standards}

Conflict of interest The authors declare that they have no conflict of interest.

Publisher's note Springer Nature remains neutral with regard to jurisdictional claims in published maps and institutional affiliations.

Open Access This article is licensed under a Creative Commons Attribution 4.0 International License, which permits use, sharing, adaptation, distribution and reproduction in any medium or format, as long as you give appropriate credit to the original author(s) and the source, provide a link to the Creative Commons license, and indicate if changes were made. The images or other third party material in this article are included in the article's Creative Commons license, unless indicated otherwise in a credit line to the material. If material is not included in the article's Creative Commons license and your intended use is not permitted by statutory regulation or exceeds the permitted use, you will need to obtain permission directly from the copyright holder. To view a copy of this license, visit http://creativecommons. org/licenses/by/4.0/.

\section{References}

1. Liu Y, Shao ML, Wu Y, Yan C, Jiang S, Liu JN, et al. Role for the endoplasmic reticulum stress sensor IRE1 alpha in liver regenerative responses. J Hepatol. 2015;62:590-8.

2. Woolbright BL, Jaeschke H. Role of the inflammasome in acetaminophen-induced liver injury and acute liver failure. J Hepatol. 2017;66:836-48.

3. Ramaiah SK, Jaeschke H. Role of neutrophils in the pathogenesis of acute inflammatory liver injury. Toxicol Pathol. 2007;35: 757-66.

4. Radi R. Nitric oxide, oxidants, and protein tyrosine nitration. PNAS. 2004;101:4003-8.

5. James LP, Mayeux PR, Hinson JA. Acetaminophen-induced hepatotoxicity. Drug Metab Dispos. 2003;31:1499-506.

6. Xue W, Huang J, Chen H, Zhang Y, Zhu X, Li J, et al. Histone methyltransferase G9a modulates hepatic insulin signaling via regulating HMGA1. Biochim Biophys Acta Mol Basis Dis. 2018;1864:338-46.

7. Kramer JM. Regulation of cell differentiation and function by the euchromatin histone methyltranserfases G9a and GLP. Biochem Cell Biol. 2016;94:26-32.

8. Pajaud J, Ribault C, Ben Mosbah I, Rauch C, Henderson C, Bellaud $\mathrm{P}$, et al. Glutathione transferases $\mathrm{P} 1 / \mathrm{P} 2$ regulate the timing of signaling pathway activations and cell cycle progression during mouse liver regeneration. Cell Death Dis. 2015;6:e1598.

9. Ding J, Li T, Wang X, Zhao E, Choi JH, Yang L, et al. The histone H3 methyltransferase G9A epigenetically activates the serine-glycine synthesis pathway to sustain cancer cell survival and proliferation. Cell Metab. 2013;18:896-907.

10. Klingmuller U, Bauer A, Bohl S, Nickel PJ, Breitkopf K, Dooley $\mathrm{S}$, et al. Primary mouse hepatocytes for systems biology approaches: a standardized in vitro system for modelling of signal transduction pathways. Syst Biol. 2006;153:433-47.

11. Wu Y, Shen J, Yin Z. Expression, purification and functional analysis of hexahistidine-tagged human glutathione S-transferase P1-1 and its cysteinyl mutants. Protein J. 2007;26:359-70.

12. Wan D, Liu C, Sun Y, Wang W, Huang K, Zheng L. MacroH2A1.1 cooperates with EZH2 to promote adipogenesis by regulating Wnt signaling. J Mol Cell Biol. 2017;9:325-37.

13. Eckersley-Maslin MA, Spector DL. Random monoallelic expression: regulating gene expression one allele at a time. Trends Genet. 2014;30:237-44.

14. Tachibana M, Sugimoto K, Nozaki M, Ueda J, Ohta T, Ohki M, et al. G9a histone methyltransferase plays a dominant role in euchromatic histone $\mathrm{H} 3$ lysine 9 methylation and is essential for early embryogenesis. Genes Dev. 2002;16:1779-91.

15. Arvelo MB, Cooper JT, Longo C, Daniel S, Grey ST, Mahiou J, et al. A20 protects mice from D-galactosamine/lipopolysaccharide acute toxic lethal hepatitis. Hepatology. 2002;35:535-43.

16. Leung TM, Lu Y, Yan W, Moron-Concepcion JA, Ward SC, Ge $\mathrm{X}$, et al. Argininosuccinate synthase conditions the response to acute and chronic ethanol-induced liver injury in mice. Hepatology. 2012;55:1596-609.

17. Blanchard-Fillion B, Prou D, Polydoro M, Spielberg D, Tsika E, Wang Z, et al. Metabolism of 3-nitrotyrosine induces apoptotic death in dopaminergic cells. J Neurosci. 2006;26:6124-30.

18. Hinson JA, Reid AB, McCullough SS, James LP. Acetaminophen-induced hepatotoxicity: role of metabolic activation, reactive oxygen/nitrogen species, and mitochondrial permeability transition. Drug Metab Rev. 2004;36:805-22.

19. Ishida Y, Kondo T, Kimura A, Tsuneyama K, Takayasu T, Mukaida N. Opposite roles of neutrophils and macrophages in the pathogenesis of acetaminophen-induced acute liver injury. Eur J Immunol. 2006;36:1028-38.

20. Elsby R, Kitteringham NR, Goldring CE, Lovatt CA, Chamberlain $\mathrm{M}$, Henderson $\mathrm{CJ}$, et al. Increased constitutive c-Jun $\mathrm{N}$ terminal kinase signaling in mice lacking glutathione S-transferase Pi. J Biol Chem. 2003;278:22243-9.

21. Lu H, Lei X, Zhang Q. Liver-specific knockout of histone methyltransferase G9a impairs liver maturation and dysregulates inflammatory, cytoprotective, and drug-processing genes. Xenobiotica. 2019;49:740-52.

22. Andrukhova O, Salama M, Krssak M, Wiedemann D, ElHousseiny L, Hacker M, et al. Single-dose GSTP1 prevents infarction-induced heart failure. J Card Fail. 2014;20:135-45.

23. Bartolini D, Commodi J, Piroddi M, Incipini L, Sancineto L, Santi $\mathrm{C}$, et al. Glutathione S-transferase pi expression regulates the Nrf2-dependent response to hormetic diselenides. Free Radic Biol Med. 2015;88:466-80.

24. Nault JC, Rebouissou S, Zucman Rossi J. NRF2/KEAP1 and Wnt/ beta-catenin in the multistep process of liver carcinogenesis in humans and rats. Hepatology. 2015;62:677-9. 
25. Irifuku T, Doi S, Sasaki K, Doi T, Nakashima A, Ueno T, et al. Inhibition of H3K9 histone methyltransferase G9a attenuates renal fibrosis and retains klotho expression. Kidney Int. 2016;89:147-57.

26. Rickels R, Herz HM, Sze CC, Cao K, Morgan MA, Collings CK, et al. Histone H3K4 monomethylation catalyzed by Trr and mammalian COMPASS-like proteins at enhancers is dispensable for development and viability. Nat Genet. 2017;49:1647-53.

27. Tachibana M, Matsumura Y, Fukuda M, Kimura H, Shinkai Y. G9a/GLP complexes independently mediate H3K9 and DNA methylation to silence transcription. EMBO J. 2008;27:2681-90.

28. Park SE, Yi HJ, Suh N, Park YY, Koh JY, Jeong SY, et al. Inhibition of EHMT2/G9a epigenetically increases the transcription of Beclin-1 via an increase in ROS and activation of NFkappaB. Oncotarget 2016;7:39796-808.

29. El Gazzar M, Yoza BK, Chen X, Hu J, Hawkins GA, McCall CEG9a. and HP1 couple histone and DNA methylation to TNFalpha transcription silencing during endotoxin tolerance. J Biol Chem. 2008;283:32198-208.

30. Fang TC, Schaefer U, Mecklenbrauker I, Stienen A, Dewell S, Chen MS, et al. Histone H3 lysine 9 di-methylation as an epigenetic signature of the interferon response. J Exp Med. 2012;209:661-9.
31. Zhang X, Peng D, Xi Y, Yuan C, Sagum CA, Klein BJ, et al. G9amediated methylation of ERalpha links the PHF20/MOF histone acetyltransferase complex to hormonal gene expression. Nat Commun. 2016;7:10810.

32. Luo L, Wang Y, Feng Q, Zhang H, Xue B, Shen J, et al. Recombinant protein glutathione S-transferases P1 attenuates inflammation in mice. Mol Immunol. 2009;46:848-57.

33. Henderson CJ, McLaren AW, Moffat GJ, Bacon EJ, Wolf CR. Piclass glutathione S-transferase: regulation and function. Chem Biol Interact. 1998;111-112:69-82.

34. Conklin DJ, Haberzettl P, Jagatheesan G, Baba S, Merchant ML, Prough RA, et al. Glutathione S-transferase $\mathrm{P}$ protects against cyclophosphamide-induced cardiotoxicity in mice. Toxicol Appl Pharm. 2015;285:136-48.

35. Conklin DJ, Haberzettl P, Lesgards JF, Prough RA, Srivastava S, Bhatnagar A. Increased sensitivity of glutathione S-transferase Pnull mice to cyclophosphamide-induced urinary bladder toxicity. $\mathrm{J}$ Pharmacol Exp Ther. 2009;331:456-69.

36. Henderson CJ, Ritchie KJ, McLaren A, Chakravarty P, Wolf CR. Increased skin papilloma formation in mice lacking glutathione transferase GSTP. Cancer Res. 2011;71:7048-60. 